\title{
THE NONLINEAR GEOMETRY OF LINEAR PROGRAMMING. III PROJECTIVE LEGENDRE TRANSFORM COORDINATES AND HILBERT GEOMETRY
}

\author{
J. C. LAGARIAS
}

\begin{abstract}
This paper studies projective scaling trajectories, which are the trajectories obtained by following the infinitesimal version of Karmarkar's linear programming algorithm. A nonlinear change of variables, projective Legendre transform coordinates, is introduced to study these trajectories. The projective Legendre transform mapping has a coordinate-free geometric interpretation in terms of the notion of "centering by a projective transformation." Let $\mathrm{H}$ be a set of linear programming constraints $\left\{\left\langle\mathbf{a}_{j}, \mathbf{x}\right\rangle \geq b_{j}: 1 \leq j \leq m\right\}$ on $\mathbf{R}^{n}$ such that its polytope of feasible solutions $P_{\mathrm{H}}$ is bounded and contains $\mathbf{0}$ in its interior. The projective Legendre transform mapping $\psi_{\mathrm{H}}$ is given by

$$
\psi_{\mathbf{H}}(\mathbf{x})=\frac{\phi_{\mathbf{H}}(\mathbf{x})}{m+\left\langle\phi_{\mathbf{H}}(\mathbf{x}), \mathbf{x}\right\rangle}, \quad \text { where } \phi_{\mathbf{H}}(\mathbf{x})=-\sum_{j=1}^{m} \frac{\mathbf{a}_{j}}{\left\langle\mathbf{a}_{j}, \mathbf{x}\right\rangle-b_{j}} .
$$

Here $\phi_{\mathbf{H}}(x)$ is the Legendre transform coordinate mapping introduced in part II. $\psi_{H}(\mathbf{x})$ is a one-to-one and onto mapping of the interior of the feasible solution polytope $\operatorname{Int}\left(P_{\mathrm{H}}\right)$ to the interior of its polar polytope $\operatorname{Int}\left(P_{\mathrm{H}}^{\circ}\right)$. The set of projective scaling trajectories with objective function $\langle\mathbf{c}, \mathbf{x}\rangle-c_{0}$ are mapped under $\psi_{\mathrm{H}}$ to the set of straight line segments in $\operatorname{Int}\left(P_{\mathrm{H}}^{\circ}\right)$ passing through the boundary point $-\mathbf{c} / c_{0}$ of $P_{\mathrm{H}}^{\circ}$. As a consequence the projective scaling trajectories (for all objective functions) can be interpreted as the complete set of "geodesics" (actually distinguished chords) of a projectively invariant metric geometry on $\operatorname{Int}\left(P_{\mathrm{H}}\right)$, which is isometric to Hilbert geometry on the interior of the polar polytope $P_{\mathrm{H}}^{\circ}$.
\end{abstract}

\section{INTRODUCTION}

This series of papers studies curves arising as trajectories of the infinitesimal analogue of two interior-point linear programming algorithms. One of these algorithms is the projective scaling algorithm (Karmarkar's algorithm), and the associated curves are called projective scaling trajectories or P-trajectories. The other is the affine scaling algorithm [B, VMF], and the associated curves are called affine scaling trajectories or A-trajectories. Part I showed that there is a simple relationship between $P$-trajectories for one linear program and the $A$ trajectories of a related linear program. Part II specified a nonlinear change of variables, Legendre transform coordinates, which linearize $A$-trajectories. The

Received by the editors February 3, 1987 and, in revised form, September 19, 1988.

1980 Mathematics Subject Classification (1985 Revision). Primary 90C05, 52A50, 49D37, 34A34; Secondary 51K99, 49D30, 90C25. 
Legendre transform mapping is given by rational functions, which implies that each $A$-trajectory is part of a real algebraic curve. Another consequence is that the set of all $A$-trajectories forms the complete set of geodesics of a metric geometry defined on the relative interior of the polytope of feasible solutions of the linear problem, and this geometry is isometric to Euclidean geometry.

This paper studies $P$-trajectories. The main tool used is a second nonlinear change of variables, projective Legendre transform coordinates. The projective Legendre transform coordinate mapping is given by rational functions, and it depends only on the constraints $H$ of the linear program and not on the objective function. The main result of the paper is that the projective Legendre transform mapping linearizes $P$-trajectories. This leads to an interpretation of the set of $P$-trajectories as "geodesics" (actually distinguished chords, see $\S 7)$ of a metric geometry on the relative interior $\operatorname{Rel}-\operatorname{Int}\left(P_{\mathrm{H}}\right)$ of the polytope $P_{\mathrm{H}}$ of feasible solutions to $\mathrm{H}$, which is isometric to Hilbert geometry on the dual polytope $P_{\mathrm{H}}^{d}$. (The dual polytope $P_{\mathrm{H}}^{d}$ is defined in $\S 2 \mathrm{~B}$.) The projective Legendre transform coordinate mapping has a simple algebraic relation to the Legendre transform coordinate mapping, which is stated in (1.4) below.

The projective Legendre transform mapping has a natural coordinate-free geometric interpretation in terms of the concept of "centering by a projective transformation," which we now explain.

Consider a set $\mathrm{H}$ of linear programming constraints on $\mathbf{R}^{n}$ in inequality form:

$$
\left\langle\mathbf{a}_{j}, \mathbf{x}\right\rangle \geq b_{j} ; \quad 1 \leq j \leq m .
$$

Let $P_{\mathrm{H}}$ denote the polytope of feasible solutions of the set of constraints $\mathrm{H}$. Throughout the paper we assume that $P_{\mathrm{H}}$ is full-dimensional, i.e. its interior $\operatorname{Int}\left(P_{\mathrm{H}}\right)$ is nonempty. (The case of lower-dimensional $P_{\mathrm{H}}$ can always be reduced to the full-dimensional case by an affine change of variable.) Associated to $\mathrm{H}$ the logarithmic barrier function $f_{\mathrm{H}}(\mathbf{x})$ given by

$$
f_{\mathrm{H}}(\mathbf{x})=-\sum_{j=1}^{m} \log \left(\left\langle\mathbf{a}_{j}, \mathbf{x}\right\rangle-b_{j}\right) .
$$

The constraints $\mathrm{H}$ are said to have a center if $f_{\mathrm{H}}(\mathbf{x})$ has a minimum on $\operatorname{Int}\left(P_{\mathrm{H}}\right)$. This occurs if and only if $P_{H}$ is bounded, and in that case there is a unique minimum $\mathbf{x}_{\mathrm{H}} \in \operatorname{Int}\left(P_{\mathrm{H}}\right)$ of $f_{\mathrm{H}}(\mathbf{x})$, which we call the center of $\mathrm{H}$. (This concides with the concept of "analytical center" of Sonnevend [So1, So2].) The center may be thought of as a kind of "balanced" point far away from the boundary of $P_{\mathrm{H}}$. It is an affine invariant in the sense that if $\mathbf{J}: \mathbf{R}^{n} \rightarrow \mathbf{R}^{n}$ is an invertible affine transformation, and if $\mathbf{J}(\mathbf{H})$ denotes the transformed set of constraints

$$
\left\langle\mathbf{a}_{j}, A^{-1}(\mathbf{y})\right\rangle \geq b_{j} ; \quad 1 \leq j \leq m,
$$

then

$$
\mathbf{x}_{\mathbf{J}(\mathbf{H})}=\mathbf{J}\left(\mathbf{x}_{\mathbf{H}}\right) .
$$



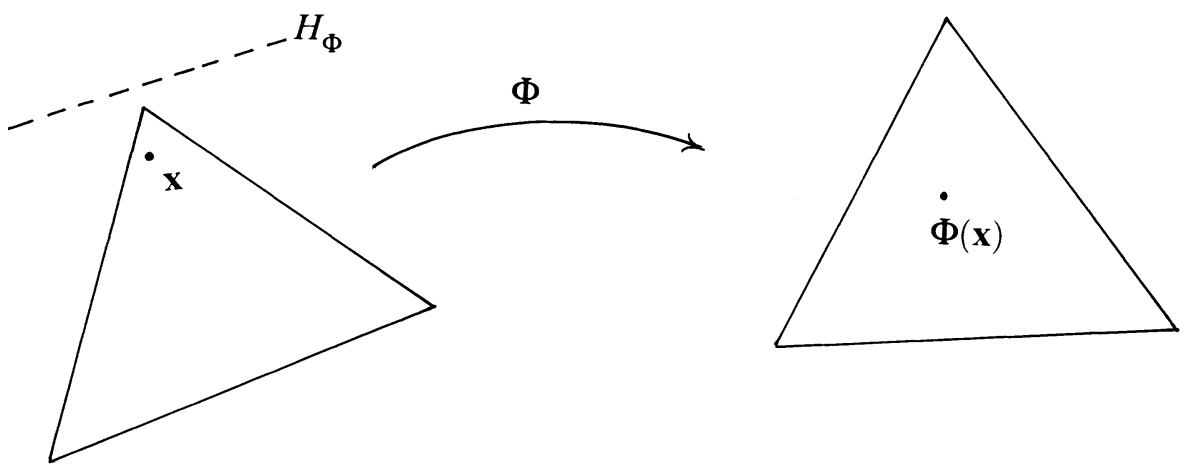

FigurE 1.1. Centering by a projective transformation

The concept of "centering by a projective transformation" is roughly as follows. Let $\Phi$ be a projective transformation. The transformed set of constraints $\Phi(H)$ is obtained from:

$$
\left\langle\mathbf{a}_{j}, \Phi^{-1}(\mathbf{y})\right\rangle \geq b_{j} ; \quad 1 \leq j \leq m,
$$

by clearing denominators to get a set of linear inequalities. We say that $\Phi$ is admissible for $\mathrm{H}$ if the polytope $P_{\Phi(\mathrm{H})}$ is bounded. We say that $\Phi$ centers $\mathbf{x}$ if $\Phi$ is admissible for $\Phi(H)$ and $\Phi(\mathbf{x})$ is the center of $\Phi(H)$. (The precise definition that $\Phi$ centers $\mathbf{x}$ requires a sign condition on the denominator of $\Phi$, in order to avoid reversing inequalities, see $\S 3$.) The notion of centering by a projective transformation is illustrated in Figure 1.1, where $H_{\Phi}$ denotes the hyperplane sent to infinity by $\Phi$.

The points near the hyperplane $H_{\Phi}$ are "stretched" more than the points far away from $H_{\Phi}$ by $\Phi$, and when $\Phi$ is properly chosen this can be done so that $\Phi(\mathbf{x})$ is the center of $\Phi(H)$. The idea of "centering by a projective transformation" is due to Karmarkar [K], though he treats only a special case, and does not give a general definition of center. This paper is apparently the first to give explicit formulae for "centering by a projective transformation" for linear programming constraints in inequality form.

The invariance property of the center under affine transformations (given by (1.2)) implies that if $\Phi$ centers $\mathbf{x}$ then so does $\mathbf{J} \circ \Phi$, where $\mathbf{J}$ is any invertible affine transformation. In $\S 3$ we prove that every point $\mathbf{x} \in \operatorname{Int}\left(P_{\mathrm{H}}\right)$ can be centered by a suitable projective transformation $\Phi$ and that the set of all projective transformations centering $\mathbf{x}$ is exactly:

$$
[\Phi]=\{\mathbf{J} \circ \Phi: \mathbf{J} \text { an invertible affine transformation }\} \text {. }
$$

which is a left coset of the affine group acting on the group of projective transformations. The hyperplane $H_{\Phi}$ mapped to the hyperplane at infinity by $\Phi$ is an invariant of the coset $[\Phi]$. The (abstract) projective Legendre transform mapping $\hat{\psi}_{H}(\mathbf{x})$ maps $\mathbf{x}$ to this hyperplane $H_{\Phi}$, where $H_{\Phi}$ is regarded as a point in dual projective space (see $\S 2 \mathrm{~A}$ ). This is the coordinate-free geometric interpretation of the projective Legendre transform coordinate map. 
For computations in affine space $\mathbf{R}^{n}$ it is convenient to use a coordinatized version of the (abstract) projective Legendre transform mapping. We choose coordinates that identify projective duality with the classical notion of polarity, as explained in $\S 2 \mathrm{C}$. In this formulation the point $\mathbf{0}$ is distinguished, and we require that the constraint set $\mathrm{H}$ have $\mathbf{0} \in \operatorname{Int}\left(P_{\mathrm{H}}\right)$. A hyperplane $H$ not containing $\mathbf{0}$ satisfies a unique linear equation of the form: $1-\langle\mathbf{a}, \mathbf{x}\rangle=0$, and is labelled $H_{\mathrm{a}}$. The set of all such hyperplanes is thereby identified with the dual space $\left(\mathbf{R}^{n}\right)^{*}$, and $\mathbf{a}=\mathbf{0}$ corresponds to the "hyperplane at infinity." With this labelling the dual polytope $P_{\mathrm{H}}^{d}$ (defined in $\S 2 \mathrm{~B}$ ) is identified with the polar polytope $P_{\mathrm{H}}^{\circ}$, where the polar body $C^{\circ}$ to a convex body $C$ containing $\mathbf{0}$ in its interior is defined by

$$
C^{\circ}=\{\mathbf{y}:\langle\mathbf{x}, \mathbf{y}\rangle \leq 1 \text { for all } \mathbf{x} \in C\} .
$$

The polar polytope $P_{\mathrm{H}}^{\circ}$ is a polytope combinatorially dual to $P_{\mathrm{H}}$ containing 0 in its interior.

The projective Legendre transform coordinate mapping $\psi_{\mathrm{H}}(\mathbf{x})$ is the abstract mapping $\hat{\psi}_{H}$ written using these coordinates. In $\S 3$ we show that it is given by

$$
\psi_{H}(\mathbf{x})=\frac{\phi_{H}(\mathbf{x})}{m+\left\langle\phi_{H}(\mathbf{x}), \mathbf{x}\right\rangle}
$$

where

$$
\phi_{\mathrm{H}}(\mathbf{x})=\nabla f_{\mathrm{H}}(\mathbf{x})=-\sum_{j=1}^{m} \frac{\mathbf{a}_{j}}{\left\langle\mathbf{a}_{j}, \mathbf{x}\right\rangle-b_{j}}
$$

is the Legendre transform coordinate mapping studied in part II. This formula for $\psi_{H}(\mathbf{x})$ shows that it is a scaled version of the Legendre transform coordinate mapping. (The scale factor $\left(m+\left\langle\phi_{\mathrm{H}}(\mathbf{x}), \mathbf{x}\right\rangle\right)^{-1}$ is positive for all $\mathbf{x} \in \operatorname{Int}\left(P_{\mathrm{H}}\right)$.) In $\S 3$ we prove that $\Psi_{H}(\mathbf{x})$ is a one-to-one and onto mapping from $\operatorname{Int}\left(P_{H}\right)$ to $\operatorname{Int}\left(P_{\mathrm{H}}^{\circ}\right)$.

In $\S 4$ we show that the abstract projective Legendre transform mapping is compatible with projective transformations. Let $\Phi^{\mathrm{PA}}$ denote the polar adjoint projective transformation to $\Phi$, a notion defined in $\S 2 \mathrm{D}$. We prove that if the projective transformation $\Phi$ is admissible for $H$ then the following diagram commutes.

$$
\begin{array}{ccc}
\operatorname{Int}\left(P_{\mathrm{H}}\right) & \stackrel{\Phi}{\rightarrow} & \operatorname{Int}\left(P_{\boldsymbol{\Phi}(\mathrm{H})}\right) \\
\psi_{\mathrm{H}} \downarrow & & \downarrow \psi_{\Phi(\mathrm{H})} \\
\operatorname{Int}\left(P_{\mathrm{H}}^{\circ}\right) & \stackrel{\Phi^{P_{A}}}{\leftarrow} & \operatorname{Int}\left(P_{\boldsymbol{\Phi}(\mathrm{H})}^{\circ}\right)
\end{array}
$$

All maps in this diagram are one-to-one and onto. This result should be compared with the set-to-set duality between polarity and adjoint projective transformation obtained by Klee [K1].

The main application of these results, proved in $\S 6$, is that the projective Legendre transform coordinate mapping linearize $P$-trajectories. More precisely, assume that $\mathbf{0} \in \operatorname{Int}\left(P_{\mathrm{H}}\right)$ and that the linear program has the objective 

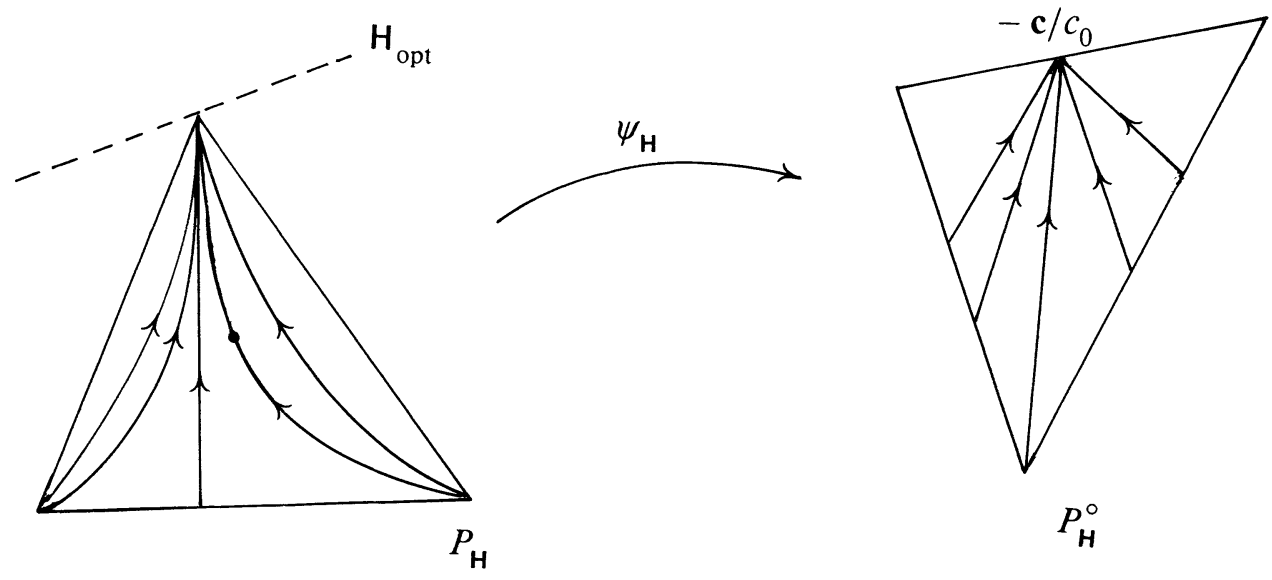

FIGURE 1.2. Effect of projective Legendre transform coordinate map $\psi_{\mathrm{H}}$ on $P$-trajectories for a normalized objective function

function

$$
\operatorname{minimize}\langle\mathbf{c}, \mathbf{x}\rangle-c_{0} \text {. }
$$

An objective function is normalized for a set of constraints $\mathrm{H}$ if $\langle\mathbf{c}, \mathbf{x}\rangle \geq c_{0}$ for all $\mathbf{x} \in P_{\mathrm{H}}$ and $\langle\mathbf{c}, \mathbf{x}\rangle=c_{0}$ for some $x \in P_{\mathrm{H}}$. Karmarkar's algorithm applies only to normalized objective functions, but it is possible to define $P$ trajectories for all objective functions, whether normalized or not, see part I. We prove that the set of $P$-trajectories for this objective function are mapped by $\psi_{H}$ to a set of line segments in $\operatorname{Int}\left(P_{\mathrm{H}}^{\circ}\right)$. If $c_{0} \neq 0$ this is the set of line segments which pass throught the point $-\mathbf{c} / c_{0}$ in $\left(\mathbf{R}^{n}\right)^{*}$ which corresponds to the hyperplane $H=\left\{\mathbf{x}:\langle\mathbf{c}, \mathbf{x}\rangle=c_{0}\right\}$, while if $c_{0}=0$ it is a family of parallel line segments in the direction $\mathbf{c}$. If the objective function is normalized, then the point $\mathrm{c} / c_{0}$ is on the boundary $\partial P_{\mathrm{H}}^{\circ}$ of the polar polytope $P_{\mathrm{H}}^{\circ}$. The main result for a normalized objective function is pictured in Figure 1.2.

This result has several consequences. First, since the projective Legendre transform coordinate mapping is a rational mapping, it directly implies that each $P$-trajectory is part of a real algebraic curve, since algebraic mappings map algebraic curves to algebraic curves, and the mapping $\psi_{\mathrm{H}}^{-1}$ is algebraic.

A second consequence is that there is a natural geometric interpretation of $P$-trajectories as "geodesics," which is discussed in $\S 7$. The geometry is a metric geometry pulled back using $\psi_{\mathrm{H}}$ from Hilbert geometry on the interior of the polar polytope $\operatorname{Int}\left(P_{\mathrm{H}}^{\circ}\right)$. The Hilbert metric $d_{C}$ for a bounded convex body $C$ in $\mathbf{R}^{n}$ is defined by

$$
d_{\mathrm{H}}\left(\mathbf{x}_{1}, \mathbf{x}_{2}\right)=\log \left(\chi\left(\mathbf{y}_{1}, \mathbf{x}_{1}, \mathbf{x}_{2}, \mathbf{y}_{2}\right)\right)
$$

where the line segment through $\mathbf{x}_{1}$ and $\mathbf{x}_{2}$ hits the boundary $\partial C$ in points 
$\mathbf{y}_{1}, \mathbf{y}_{2}$, in the order $\left(\mathbf{y}_{1}, \mathbf{x}_{1}, \mathbf{x}_{2}, \mathbf{y}_{2}\right)$, and

$$
\chi\left(\mathbf{y}_{1}, \mathbf{x}_{1}, \mathbf{x}_{2}, \mathbf{y}_{2}\right)=\frac{\left\|\mathbf{y}_{1}-\mathbf{x}_{2}\right\|\left\|\mathbf{x}_{1}-\mathbf{y}_{2}\right\|}{\left\|\mathbf{y}_{1}-\mathbf{x}_{1}\right\|\left\|\mathbf{x}_{2}-\mathbf{y}_{2}\right\|}
$$

is the cross-ratio of these points. Hilbert showed that $d_{\mathrm{H}}$ is a metric, and that straight line segments are curves of shortest distance between two points. Such curves are called extremals. In the case that $C$ is a polytope, there are extremals which are not straight line segments, so that geodesics are not defined in the usual sense. Busemann [Bu2] introduced a notion of a distinguished chord which generalizes the notion of geodesic to the case of non-unique extremals. The set of straight line segments in $C$ always form a complete set of distinguished chords in Busemann's sense. Thus one obtains a metric $d_{\mathrm{H}}(\cdot, \cdot)$ on $\operatorname{Int}\left(P_{\mathrm{H}}\right)$ which is the pullback under $\psi_{\mathrm{H}}$ of the Hilbert metric on $\operatorname{Int}\left(P_{\mathrm{H}}^{\circ}\right)$, for which $P$-trajectories (for all normalized objective functions) form a complete set of distinguished chords. This metric is invariant under projective transformations in the sense that

$$
d_{H}\left(\mathbf{x}_{1}, \mathbf{x}_{2}\right)=d_{\Phi(H)}\left(\Phi\left(\mathbf{x}_{1}\right), \Phi_{2}\left(\mathbf{x}_{2}\right)\right) .
$$

Hilbert geometry has proved useful in understanding several problems in convexity and optimization, cf. [Bush 1, Bush $2, \mathrm{Bi}, \mathrm{KP}$ ]. The metric $d_{\mathrm{H}}$ provides a projective invariant with which to measure the performance of Karmarkar's algorithm and related algorithms, and it may be useful in obtaining improved running time bounds for such algorithms.

A third consequence is that projective Legendre transform coordinates can be used to study the asymptotic behavior of $P$-trajectories. This gives an alternative to the approach of Shub [Sh], and has the advantage that it applies to degenerate linear programs. Details will be given elsewhere.

The projective Legendre transform coordinate mapping uses the notion of projective duality, which is distinct from the notion of dual linear program in the theory of linear programming. In $\S 8$ we describe a relation between these two concepts of duality. There is a rational mapping from the interior of the polytope of dual feasible solutions into the interior of the polytope polar to the primal feasible solution polytope. This mapping is compatible with the projective Legendre transform coordinate mapping for those points on the central $P$-trajectory, which is the $P$-trajectory through the center of the primal feasible solution polytope.

The contents of the paper are as follows. $\S 2$ reviews basic results on projective transformations, projective duality and polarity. $\S 3$ defines the notion of centering by a projective transformation and constructs the projective Legendre transform coordinate mapping. $\S 4$ shows that the projective Legendre transform mapping is compatible with projective transformations. $\S 5$ defines the projective scaling direction field and $P$-trajectories for a linear program with inequality constraints (1.1). We remark that the definition of $P$-trajectory in part I was given for linear programs in Karmarkar's canonical form, which have equality constraints. The definition given in $\S 5$ agrees with this definition under 
an affine transformation. (This equivalence is described in more detail in [BL].) $\S 6$ shows that $P$-trajectories are linearized in projective Legendre transform coordinates. $\S 7$ discusses Hilbert geometry and the metric $d_{\mathrm{H}} \cdot \S 8$ establishes relations between linear programming duality and projective duality.

\section{Projective tRansformations, POLAR POLYTOPES,} AND PROJECTIVE DUALITY

This section describes basic facts about affine and projective transformations. Vectors in the space $\mathbf{R}^{n}, \mathbf{P}^{n}$ are column vectors, while vectors in the dual spaces $\left(\mathbf{R}^{n}\right)^{*},\left(\mathbf{P}^{n}\right)^{*}$ are row vectors.

A. Projective space and affine space. Projective space $\mathbf{P}^{n}$ is the set of lines in $\mathbf{R}^{n+1}$ passing through the origin. A projective point $[\mathbf{x}]$ is given by

$$
[\mathbf{x}]=\left\{\lambda\left(x_{0}, x_{1}, \ldots, x_{n}\right)^{T}: \lambda \neq 0 \text { and some } x_{i} \neq 0\right\} .
$$

The coordinates $\mathbf{x} \in \mathbf{R}^{n+1}$ are called homogeneous coordinates. Projective space $\mathbf{P}^{n}$ has a metrizable topology with metric

$$
d\left(\left[\mathbf{x}_{1}\right],\left[\mathbf{x}_{2}\right]\right)=\sqrt{2-\frac{2\left|\left\langle\mathbf{x}_{1}, \mathbf{x}_{2}\right\rangle\right|}{\left\|\mathbf{x}_{1}\right\|\left\|\mathbf{x}_{2}\right\|}},
$$

which measures the shorter of the chordal distances between the points $\pm \mathbf{x}_{1} /\left\|\mathbf{x}_{1}\right\|$ and $\pm \mathbf{x}_{2} /\left\|\mathbf{x}_{2}\right\|$ on the unit sphere. With this topology $\mathbf{P}^{n}$ is compact.

By affine space we mean $\mathbf{R}^{n}$ with its usual topology, with a notion of straight line and incidence of lines, but without the Euclidean metric structure, so that there are no notions of distance or angle. We regard projective space $\mathbf{P}^{n}$ as being affine space with some "points of infinity" adjoined. More precisely, we imbed $\mathbf{R}^{n}$ in $\mathbf{P}^{n}$ by

$$
\mathbf{x}=\left(x_{1}, \ldots, x_{n}\right)^{T} \in \mathbf{R}^{n} \rightarrow\left[\left(1, x_{1}, x_{2}, \ldots, x_{n}\right)^{T}\right] \in \mathbf{P}^{n} .
$$

Thus $\mathbf{R}^{n}$ is identified with the set of projective points whose first coordinate does not vanish. The set $H_{\infty}^{P}=\left\{[\mathbf{x}] \in \mathbf{P}^{n}: x_{0}=0\right\}$ is called the (projective) hyperplane at infinity and is isomorphic to $\mathbf{P}^{n-1}$, and $\mathbf{R}^{n}=\mathbf{P}^{n}-\mathbf{H}_{\infty}^{P}$. We call the embedding (2.2) the standard embedding of $\mathbf{R}^{n}$ in $\mathbf{P}^{n}$, and use it to regard $\mathbf{R}^{n}$ as a subset of $\mathbf{P}^{n}$.

A projective hyperplane $H$, or p-hyperplane, is a set of points in $\mathbf{P}^{n}$ satisfying a homogeneous linear equation:

$$
H_{\mathrm{a}}^{P}=\left\{[\mathbf{x}]: \sum_{i=0}^{n} a_{i} x_{i}=0\right\}
$$

where $\mathbf{a}=\left(a_{1}, \ldots, a_{n}\right)^{T} \neq \mathbf{0}$. A projective hyperplane is linearly isomorphic to the projective space $\mathbf{P}^{n-1}$. Let $\mathbf{P} H^{n}$ denote the set of all projective hyperplanes.

There is a duality between points and hyperplanes in $\mathbf{P}^{n}$. We associate to the $p$-hyperplane $H_{\mathbf{a}}$ the point [a] in the dual projective space $\left(\mathbf{P}^{n}\right)^{*}$. This is 
well defined since $H_{\mathbf{a}}=H_{\lambda \mathbf{a}}$ if $\lambda \neq 0$. A point $[\mathbf{x}]$ is on a $p$-hyperplane $H_{\mathbf{a}}$ if and only if

$$
\langle\mathbf{a}, \mathbf{x}\rangle=\sum_{i=0}^{n} a_{i} x_{i}=0 .
$$

We use row vectors a to denote homogeneous coordinates for $\left(\mathbf{P}^{n}\right)^{*}$, and call the correspondence

$$
\mathbf{a} \in\left(\mathbf{P}^{n}\right)^{*} \stackrel{d}{\leftrightarrow} H_{\mathbf{a}} \in \mathbf{P} H^{n}
$$

the duality correspondence.

There is an imperfect duality between points and (affine) hyperplanes in Euclidean space $\mathbf{R}^{n}$. To obtain $\mathbf{R}^{n}$ from $\mathbf{P}^{n}$ one removes one (projective) hyperplane from $\mathbf{P}^{n}$, the hyperplane at infinity. This destroys the symmetry between points and hyperplanes, Sinden [S2] observes that one can restore symmetry in the affine situation by singling out a special point, which we may suppose is $\mathbf{0}$. Any hyperplane $H$ in $\mathbf{R}^{n}$ not passing through $\mathbf{0}$ has a unique expression as

$$
H_{\mathbf{a}}=\left\{\mathbf{x}: 1+\sum_{i=1}^{n} a_{i} x_{i}=0\right\},
$$

and we identify such a hyperplane $H_{\mathrm{a}}$ with the point a lying in the dual space $\left(\mathbf{R}^{n}\right)^{*}$. We represent points in the dual space $\left(\mathbf{R}^{n}\right)^{*}$ as row vectors. The dual space $\left(\mathbf{R}^{n}\right)^{*}$ is embedded in the dual projective space $\left(\mathbf{P}^{n}\right)^{*}$ by the standard embedding

$$
\left(a_{1}, \ldots, a_{n}\right) \in\left(\mathbf{R}^{n}\right)^{*} \rightarrow\left[\left(1, a_{1}, \ldots, a_{n}\right)\right] \in\left(\mathbf{P}^{n}\right)^{*} .
$$

A projective line or a $p$-line, $l$, is the set of all linear combinations of the distinct projective points $\left[\mathbf{x}_{1}\right]$ and $\left[\mathbf{x}_{2}\right]$ :

$$
l=\left\{\left[\alpha_{1} \mathbf{x}_{1}+\alpha_{2} \mathbf{x}_{2}\right]: \alpha_{1}, \alpha_{2} \in \mathbf{R},\left(\alpha_{1}, \alpha_{2}\right) \neq(0,0)\right\} .
$$

A projective line is linearly isomorphic to $\mathbf{P}^{1}$.

A projective transformation $\Phi: \mathbf{P}^{n} \rightarrow \mathbf{P}^{n}$ is defined by

$$
\Phi(x)=\Phi_{A}(\mathbf{x})=[A \mathbf{x}]
$$

where $\mathbf{x} \mapsto A \mathbf{x}$ is an invertible homogeneous linear mapping on $\mathbf{R}^{n+1}$. This is well defined, and $\Phi_{A}=\Phi_{\lambda A}$ for any $\lambda \neq 0$. A projective transformation is a homeomorphism (for the usual topology on projective space) and maps $p$-lines to $p$-lines. All projective transformations are invertible, with inverse given by $\Phi^{-1}(\mathbf{x})=\left[A^{-1} \mathbf{x}\right]$. The set $G \mathbf{P}(n, \mathbf{R})$ of all projective transformations forms a group under composition of operations. One can easily show that $\Phi_{A} \equiv \Phi_{B}$ implies that $B=\lambda A$ for some nonzero scalar $\lambda$, so that

$$
G \mathbf{P}(n, \mathbf{R}) \cong G L(n+1, \mathbf{R}) / \mathbf{R}^{*}
$$

where $\mathbf{R}^{*}=\mathbf{R}-\{\mathbf{0}\}$. 
By a projective transformation $\Phi$ on affine space $\mathbf{R}^{n}$ we mean the restriction to $\mathbf{R}^{n}$ of a projective transformation on $\mathbf{P}^{n}$. Let $\Phi(\mathbf{x})=A \mathbf{x}$ be the projective transformation on $\mathbf{P}^{n}$ with

$$
A=\left[\begin{array}{cccc}
a_{00} & a_{01} & \ldots & a_{0 n} \\
\vdots & & & \vdots \\
a_{n 0} & a_{n 1} & \ldots & a_{n n}
\end{array}\right]=\left[\begin{array}{cc}
a_{00} & \mathbf{a}_{0} \\
a_{10} & \mathbf{a}_{1} \\
\vdots & \vdots \\
a_{n 0} & \mathbf{a}_{n}
\end{array}\right] .
$$

This projective transformation $\Phi$ with domain $\mathbf{R}^{n}$ has the form

$$
\Phi\left(\left(x_{1}, \ldots, x_{n}\right)^{T}\right)=\left(\frac{a_{10}+\left\langle\mathbf{a}_{1}, \mathbf{x}\right\rangle}{a_{00}+\left\langle\mathbf{a}_{0}, \mathbf{x}\right\rangle}, \ldots, \frac{a_{n 0}+\left\langle\mathbf{a}_{n}, \mathbf{x}\right\rangle}{a_{00}+\left\langle\mathbf{a}_{0}, \mathbf{x}\right\rangle}\right)
$$

whenever the right side is defined (as an element of $\mathbf{R}^{n}$ ). We call $a_{00}+\left\langle\mathbf{a}_{0}, \mathbf{x}\right\rangle$ a denominator of $\Phi$; it is well defined up to multiplication by a nonzero scalar. We say that

$$
H_{\Phi}=\left\{\mathbf{x}: a_{00}+\left\langle\mathbf{a}_{0}, \mathbf{x}\right\rangle=0\right\}
$$

is the hyperplane mapped to infinity by $\Phi$, and that $\mathbf{R}^{n}-H_{\Phi}$ is the domain of $\Phi$ (regarded as a projective transformation on $\mathbf{R}^{n}$ ). In the case that the denominator of $\Phi$ is a nonzero constant, i.e. $a_{00} \neq 0$ and $\mathbf{a}_{0}=\mathbf{0}$, then we define $H_{\Phi}$ to be the hyperplane at infinity $H_{\infty}^{P}$.

We say that $\Psi$ is an affine transformation if $\Psi$ is a projective transformation whose denominator is constant, i.e. a projective transformation that leaves the hyperplane at infinity fixed. Affine transformations $\Psi: \mathbf{R}^{n} \rightarrow \mathbf{R}^{n}$ have the form

$$
\Psi(\mathbf{x})=A \mathbf{x}+\mathbf{b}
$$

where $A$ is an $n \times n$ matrix and $\mathbf{b}$ is a column vector. Written as a projective transformation on $\mathbf{P}^{n}$ it has the form

$$
\Psi([\mathbf{x}])=[\tilde{A} \mathbf{x}]
$$

where

$$
\tilde{A}=\left[\begin{array}{cccc}
1 & 0 & \ldots & 0 \\
b_{1} & & & \\
\vdots & & A & \\
b_{n} & & &
\end{array}\right] .
$$

The set $G A(n, \mathbf{R})$ of all invertible affine transformations forms a subgroup of the projective group $G \mathbf{P}(n, \mathbf{R})$. It is not a normal subgroup of $G \mathbf{P}(n, \mathbf{R})$; this corresponds to the fact that the choice of hyperplane at infinity is arbitrary.

B. Projective convexity and duality of projectively convex sets. There is a concept of convexity for sets in projective space $\mathbf{P}^{n}$ which is compatible with the duality of lines and hyperplanes. It associates to a (projectively) convex body $C$ in $\mathbf{P}^{n}$ a dual body $C^{d}$ in the space of hyperplanes $\mathbf{P} H^{n}$ such that $C^{d d}=C$. This notion of convexity in projective space $\mathbf{P}^{n}$ differs slightly from that in affine 
space $\mathbf{R}^{n}$; it was worked out by E. Steinitz [St, part II], and is described in W. Fenchel [F2, pp. 52-56] and in Sinden [S2]. A set $C$ is projectively convex if:

(1) The interior $\operatorname{Int}(C)$ of $C$ is not empty.

(2) The closure $\bar{C}$ of $C$ is not all of $\mathbf{P}^{n}$.

(3) $C$ is connected.

(4) Through each point $p$ not in $\bar{C}$ there is a projective hyperplane disjoint from $\bar{C}$.

The concept of projective convexity coincides with the usual (affine) notion of convexity for bounded sets in $\mathbf{R}^{n}$ : A bounded set in $\mathbf{R}^{n}$ is convex if and only if it is projectively convex. However it differs from the affine notion of convexity for some unbounded sets in $\mathbf{R}^{n}$. For example a half-space $C=\{\mathbf{x}:\langle\mathbf{c}, \mathbf{x}\rangle \geq 0\}$ in $\mathbf{R}^{n}$ is not a projectively convex set, because $\bar{C}$ includes the entire projective hyperplane at infinity and one can check that all projective hyperplanes meet $\bar{C}$. In fact, a projectively convex set in $\mathbf{P}^{n}$ is exactly the image under a projective transformation of a bounded convex set in $\mathbf{R}^{n}$ that has nonempty interior.

The dual set $C^{d}$ in $\mathbf{P}^{n}$ is the set of all projective hyperplanes in $\mathbf{P}^{n}$ that have no points in common with $\bar{C}$. We may view $C^{d}$ as a subset of $\left(\mathbf{P}^{n}\right)^{*}$ using the duality correspondence (2.3). The definition of a projectively convex set $C$ implies that $C^{d}$ is nonempty and furthermore that $C^{d}$ is an open set in $\left(\mathbf{P}^{n}\right)^{*}$. The duality theory for convex bodies in projective space consequently applies to open projectively convex sets.

Theorem 2.1. Let $C$ be an open projectively convex set in $\mathbf{P}^{n}$. Then $C^{d}$ is an open projectively convex set in $\left(\mathbf{P}^{n}\right)^{*}$ and $C^{d d}=C$.

Proof. The proof in [Fe2, p. 54] carries over to this situation. See also [S2].

We may complete this projective duality theory of convex sets by defining the empty set in $\mathbf{P}^{n}$ to be dual to the set $\left(\mathbf{P}^{n}\right)^{*}$ and the set $\mathbf{P}^{n}$ to be dual to the empty set in $\left(\mathbf{P}^{n}\right)^{*}$.

We say that a set $C$ in $\mathbf{P}^{n}$ is projectively bounded if there is a $p$-hyperplane in $\mathbf{P}^{n}$ that does not meet $\bar{C}$, the closure of $C$. This is a natural extension of the notion of bounded set in $\mathbf{R}^{n}$, in two ways:

(1) A set $C$ in $\mathbf{R}^{n}$ is projectively bounded if and only if it is bounded.

(2) A set $C$ in $\mathbf{P}^{n}$ is projectively bounded if and only if it is the image $\Phi(\hat{C})$ of a bounded set $\hat{C}$ in $\mathbf{R}^{n}$ under a projective transformation $\Phi: \mathbf{R}^{n} \rightarrow \mathbf{P}^{n}$.

The definition of a projectively convex set $C$ requires that $C$ (and $C^{d}$ ) be projectively bounded. This boundedness condition is necessary in order that $C$ and $C^{d}$ both be full-dimensional sets in $\mathbf{P}^{n}$. It is possible to use a modified definition of projectively convex set that applies to some lower-dimensional sets in $\mathbf{P}^{n}$ as well, for example the notion of p-convexity defined in Fenchel [Fe2]. The definition then is

$\left(1^{\prime}\right) C$ is nonempty. 
$\left(2^{\prime}\right) C$ is not all of $\mathbf{P}^{n}$.

$\left(3^{\prime}\right) C$ is connected.

$\left(4^{\prime}\right)$ Through each point $p$ not in $C$ there is a projective hyperplane disjoint from $C$.

This is a more general notion than the one we use.

C. Polarity. The notion of projective duality for projectively convex sets is completely symmetrical; the corresponding notion for affine spaces is not. The removal of the hyperplane at infinity from $\mathbf{P}^{n}$ to give $\mathbf{R}^{n}$ must be counterbalanced by the selection of a distinguished point in $\mathbf{R}^{n}$ to obtain a duality for convex sets. We take this special point to be $\mathbf{0}$, and the projective duality of projectively convex sets is then closely related to polarity. (More precisely, this is polarity with respect to a sphere in the terminology of Fenchel [Fe2].)

The polar set $C^{\circ}$ to a convex set $C$ in $\mathbf{R}^{n}$ is defined by

$$
C^{\circ}=\left\{\mathbf{a} \in\left(\mathbf{R}^{n}\right)^{*}:\langle\mathbf{a}, \mathbf{x}\rangle \leq 1 \text { for all } \mathbf{x} \in C\right\} .
$$

This definition makes sense for any convex set, and one always has $\mathbf{0} \in C^{\circ}$ and

$$
\operatorname{Int}\left(C^{\circ}\right)=\left\{\mathbf{a} \in\left(\mathbf{R}^{n}\right)^{*}:\langle\mathbf{a}, \mathbf{x}\rangle<1 \text { for all } \mathbf{x} \in C\right\} .
$$

Using this fact one can verify for convex sets that

$$
\operatorname{Int}\left((\operatorname{Int} C)^{\circ}\right)=\operatorname{Int}\left(C^{\circ}\right) .
$$

The polarity relation involves a different correspondence between points in $\left(\mathbf{P}^{n}\right)^{*}$ and hyperplanes in $\mathbf{P}^{n}$ than the duality correspondence. It uses the $(n+1) \times(n+1)$ diagonal matrix

$$
S=\operatorname{diag}(1,-1, \ldots,-1, \ldots,-1)=\left[\begin{array}{lllll}
1 & & & & \\
& -1 & & & \\
& & \ldots & & \\
& & -1 & \\
& & & -1
\end{array}\right]
$$

which satisfies $S^{2}=I$. The polarity correspondence between points and hyperplanes is

$$
\mathbf{a} \in\left(\mathbf{P}^{n}\right)^{*} \stackrel{\circ}{\leftrightarrow} H_{S \mathbf{a}}^{P} \in \mathbf{P} H^{n} .
$$

Using the polarity correspondence applied to points $\mathbf{a} \in C^{\circ}$ we obtain

$$
\mathbf{a} \in C^{\circ} \stackrel{\circ}{\leftrightarrow} \mathrm{H}_{\infty}^{P}=\left\{[\mathbf{x}]: x_{0}=0\right\},
$$

and for $\mathbf{a} \neq \mathbf{0}$ that

$$
\mathbf{a} \in C^{\circ} \stackrel{\circ}{\leftrightarrow} H_{-\mathbf{a}}=\left\{[\mathbf{x}]: 1-\sum_{i=1}^{n} a_{i} x_{i}=0\right\} .
$$

The polarity relation for bounded convex sets $C$ in $\mathbf{R}^{n}$ coincides up to a sign with projective duality in the case that $\mathbf{0}$ is in the interior of $C$. 
Theorem 2.2. Let $C$ be an open bounded convex set in $\mathbf{R}^{n}$ containing $\mathbf{0}$. The sets $C^{d}$ and $C^{\circ}$ viewed as subsets of $\left(\mathbf{R}^{n}\right)^{*}$ are related by

$$
-C^{d}=\operatorname{Int}\left(C^{\circ}\right)=\left\{\mathbf{a} \in\left(\mathbf{R}^{n}\right)^{*}:\langle\mathbf{a}, \mathbf{x}\rangle<1 \text { for all } \mathbf{x} \in C\right\} .
$$

Proof. By definition of $C^{d}$ viewed in $\left(\mathbf{R}^{n}\right)^{*}$ we have

$$
-C^{d}=\left\{\left(-a_{1}, \ldots,-a_{n}\right): 1+\sum_{i=1}^{n} a_{i} x_{i} \neq 0 \text { for all } \mathbf{x} \in C\right\} \text {. }
$$

We check that $-C^{d} \subseteq \operatorname{Int}\left(C^{\circ}\right)$. Since $C$ is bounded the hyperplane at infinity is in $C^{d}$, which yields $\mathbf{0} \in-C^{d}$ viewed in $\left(\mathbf{R}^{n}\right)^{*}$. If $-\mathbf{a} \in-C^{d}$ and $\mathbf{0} \in C$ the condition

$$
1+\sum_{i=1}^{n} a_{i} x_{i} \neq 0 \quad \text { for all } \mathbf{x} \in C
$$

implies that

$$
1-\sum_{i=1}^{n} a_{i} x_{i}>0 \quad \text { for all } \mathbf{x} \in C
$$

Hence $\mathbf{a} \in \operatorname{Int}\left(C^{\circ}\right)$.

Conversely if $\mathbf{a} \in \operatorname{Int}\left(C^{\circ}\right)$ then (2.13) holds so $1-\sum_{i=0}^{n} a_{i} x_{i} \neq 0$ for all $\mathbf{x} \in C$ and so $\operatorname{Int}\left(C^{\circ}\right) \subseteq C^{d}$.

The correspondence between polar sets and projectively dual convex sets breaks down if $\mathbf{0}$ is not in $C$.

In the sequel we will use polarity only in the case that $C$ is a closed bounded polytope containing $\mathbf{0}$ in its interior. In that case the following facts hold.

Theorem 2.3. Let $P$ be a closed bounded polytope containing $\mathbf{0}$ in its interior and $P^{\circ \circ}=P$. The polytope $P^{\circ}$ is combinatorially dual to $P$, i.e., its face lattice is dual to the face lattice of $P$. The points on the boundary $\partial P^{\circ}=P^{\circ}-\operatorname{Int}\left(P^{\circ}\right)$ correspond to the supporting hyperplanes of $P$.

Proof. All statements except the last are proved in [SW, pp. 76-77]. The last fact follows by observing that $\mathbf{a} \in \partial P^{\circ} \Leftrightarrow \mathbf{a} \in P^{\circ}$ and there exists $\mathbf{x} \in P$ with $\langle\mathbf{a}, \mathbf{x}\rangle=1$. It is clear from the definition (2.6) that $H_{\mathbf{a}}=\{\mathbf{x}:\langle\mathbf{a}, \mathbf{x}\rangle=1\}$ is a supporting hyperplane of $C$.

D. Adjoint projective transformations and polarity. There is a relation between adjoint projective transformations and polarity formulated by $\mathrm{V}$. Klee [K1]. We describe a version of it adapted to the projective duality correspondence and then state Klee's version of it for the polarity correspondence.

Associate to each projective transformation $\Phi: \mathbf{P}^{n} \rightarrow \mathbf{P}^{n}$ defined by $\Phi([\mathbf{x}])=$ $[A \mathbf{x}]$ its adjoint projective transformation $\Phi^{A}:\left(\mathbf{P}^{n}\right)^{*} \rightarrow\left(\mathbf{P}^{n}\right)^{*}$ defined by

$$
\Phi^{A}([\mathbf{a}])=[\mathbf{a} A] .
$$


It is clear that

$$
\left(\Phi^{A}\right)^{A} \equiv \Phi
$$

The duality correspondence between points $\mathbf{a}$ in $\left(\mathbf{P}^{n}\right)^{*}$ and hyperplanes in $\mathbf{P}^{n}$ is compatible with projective transformations in the following sense.

Lemma 2.4. Let $\Phi([\mathbf{x}])=\left[\right.$ Ax] be a projective transformation and $\Phi^{A}$ its adjoint. Then the following diagram commutes:

$$
\begin{array}{ccc}
H_{\mathbf{a}}^{P} & \stackrel{\Phi}{\longrightarrow} & H_{\mathbf{a} A^{-1}}^{P} \\
d \uparrow & & d \uparrow \\
{[\mathbf{a}]} & \stackrel{\Phi^{A}}{\leftrightarrows} & \mathbf{a} A^{-1}
\end{array}
$$

Proof. If $\Phi([\mathbf{x}])=[A \mathbf{x}]$ then $\Phi^{-1}([\mathbf{y}])=\left[A^{-1} \mathbf{y}\right]$ hence

$$
\begin{aligned}
\Phi\left(H_{\mathbf{a}}^{P}\right) & =\{\Phi([\mathbf{x}]):\langle\mathbf{a}, \mathbf{x}\rangle=0\} \\
& =\left\{\mathbf{y}:\left\langle\mathbf{a}, A^{-1} \mathbf{y}\right\rangle=0\right\} \\
& =\left\{\mathbf{y}:\left\langle\mathbf{a} A^{-1}, \mathbf{y}\right\rangle=0\right\}=H_{\mathbf{a} A^{-1}}^{P}
\end{aligned}
$$

Hence $\Phi^{A}\left(\mathbf{a} A^{-1}\right)=\left[\left(\mathbf{a} A^{-1}\right) A\right]=[\mathbf{a}]$.

This result has an immediate consequence for projectively dual convex sets.

Theorem 2.5. Let $C$ be an open, projectively convex set, and let $\Phi: \mathbf{P}^{n} \rightarrow \mathbf{P}^{n}$ be a projective transformation. Then $\Phi(C)$ is an open projectively convex set and

$$
\Phi^{A}\left(\Phi(C)^{d}\right)=C^{d} \text {. }
$$

This result asserts that the following diagram commutes in the sense given by $(2.17)$, where the vertical arrows indicate projective duality for projectively convex sets

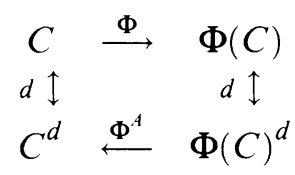

Proof. Let $\mathbf{a} \in C^{d}$. Then $H_{\mathbf{a}} \cap \bar{C}=\varnothing$, so that $H_{\mathbf{a}^{\prime}}=\Phi\left(H_{\mathrm{a}}\right)$ has $H_{\mathbf{a}^{\prime}} \cap \overline{\Phi(C)}=$ $\varnothing$. Hence $\mathbf{a}^{\prime} \in \Phi(C)^{d}$ and by (2.16) $\Phi^{A}\left(\mathbf{a}^{\prime}\right)=\mathbf{a}$. Hence $C^{d} \subseteq \Phi^{A}\left(\Phi(C)^{d}\right)$. If $\mathbf{a}^{\prime} \in \Phi(C)^{d}$ then $H_{\mathbf{a}^{\prime}} \subset \overline{\Phi(C)}=\varnothing$ so $H_{\mathbf{a}} \cap \bar{C}=\varnothing$ where $H_{\mathbf{a}}=\Phi^{-1}\left(H_{\mathbf{a}^{\prime}}\right)$. Thus $\mathbf{a} \in C^{d}$ and by $(2.16) \Phi^{A}\left(\mathbf{a}^{\prime}\right)=\mathbf{a}$. So $\Phi^{A}\left(\Phi(C)^{d}\right) \subseteq C^{d}$.

There is a similar set of facts for projective transformations $\Phi$ restricted to $\mathbf{R}^{n}$, where duality is replaced by polarity. This is due to Klee [K1]. We must use a modified notion of adjoint because the polarity correspondence differs from the duality correspondence. We define the polar adjoint $\Phi^{P A}:\left(\mathbf{P}^{n}\right)^{*} \rightarrow\left(\mathbf{P}^{n}\right)^{*}$ of a projective transformation $\Phi([\mathbf{x}])=[A \mathbf{x}]$ by

$$
\Phi^{P A}([\mathbf{a}])=[\mathbf{a} S A S],
$$


where $S=\operatorname{diag}(1,-1,-1, \ldots,-1)$. Viewing this in the affine space $\mathbf{R}^{n}$ we consider $\mathbf{0}$ as a distinguished point and only consider projective transformations $\Phi$ that do not send 0 to infinity. In this case the denominator of $\Phi$ may be scaled (uniquely) to have the form $1-\langle\mathbf{c}, \mathbf{x}\rangle$, and we may write $\Phi(\mathbf{x})=[A \mathbf{x}]$, where

$$
A=\left[\begin{array}{cc}
1 & -\mathbf{c} \\
\mathbf{b} & \tilde{A}
\end{array}\right] \text {. }
$$

Viewing this on $\mathbf{R}^{n}$ we have

$$
\Phi(\mathbf{x})=\frac{\tilde{A} \mathbf{x}+\mathbf{b}}{1-\langle\mathbf{c}, \mathbf{x}\rangle} \quad \text { for } \mathbf{x} \in \mathbf{R}^{n},
$$

and a simple computation gives

$$
\Phi^{P A}(\mathbf{a})=\frac{\mathbf{a} \tilde{A}+\mathbf{c}}{1-\langle\mathbf{a}, \mathbf{b}\rangle} \quad \text { for } \mathbf{a} \in\left(\mathbf{R}^{n}\right)^{*} .
$$

Now define

$$
\begin{aligned}
& \operatorname{domain}(\Phi)=\left\{\mathbf{x} \in \mathbf{R}^{n}:\langle\mathbf{c}, \mathbf{x}\rangle<1\right\} \\
& \operatorname{range}(\Phi)=\left\{\mathbf{y} \in \mathbf{R}^{n}: \mathbf{y}=\Phi(\mathbf{x}), \mathbf{x} \in \operatorname{domain}(\Phi)\right\}
\end{aligned}
$$

and similarly for $\Phi^{P A}$.

Theorem 2.6. If $\Phi: \mathbf{R}^{n} \rightarrow \mathbf{R}^{n}$ and $\Phi^{A}:\left(\mathbf{R}^{n}\right)^{*} \rightarrow\left(\mathbf{R}^{n}\right)^{*}$ are adjoint projective transformations, then

(i) For all $\mathbf{x} \in \operatorname{domain}(\Phi)$ and $\mathbf{a} \in \operatorname{domain}\left(\Phi^{P A}\right)$

$$
\langle\mathbf{a}, \Phi(\mathbf{x})\rangle<1 \Leftrightarrow\left\langle\Phi^{P A}(\mathbf{a}), \mathbf{x}\right\rangle<1 \text {. }
$$

(ii) For each set $W \subseteq$ domain $(\Phi)$

$$
\Phi^{P A}\left(\Phi(W)^{\circ}\right) \cap \operatorname{domain}\left(\Phi^{P A}\right)=W^{\circ} \cap \operatorname{range}\left(\Phi^{P A}\right) .
$$

Proof. This is [K1, Theorem 1].

This theorem asserts that the following diagram commutes, where the mappings are all interpreted as set-to-set mappings.

$$
\begin{array}{ccc}
W & \stackrel{\Phi}{\leftrightarrow} & \Phi(W) \\
\circ \uparrow & & \circ \downarrow \\
W^{\circ} & \stackrel{\Phi^{P A}}{\leftrightarrow} & \Phi(W)^{\circ}
\end{array}
$$

E. The left coset space $G A(n, \mathbf{R}) \backslash G \mathbf{P}(n, \mathbf{R})$ and the dual projective space $\left(\mathbf{P}^{n}\right)^{*}$. There is a natural identification of the left coset space $G A(n, \mathbf{R}) \backslash G \mathbf{P}(n, \mathbf{R})$ with the space $\mathbf{P} H^{n}$ of hyperplanes in $\mathbf{P}^{n}$. We regard affine space $\mathbf{R}^{n}$ as embedded in projective space $\mathbf{P}^{n}$ with the standard embedding. We define a mapping $\eta_{H}: G \mathbf{P}(n, \mathbf{R}) \rightarrow \mathbf{P} H^{n}$, which we call the hyperplane mapping, by

$$
\eta_{H}(\Phi)=H_{\Phi} \equiv \Phi^{-1}\left(H_{\infty}\right)
$$

where $H_{\infty}$ is the projective hyperplane at infinity, i.e. $\eta_{H}(\Phi)$ is the (projective) hyperplane mapped to infinity by $\Phi$. 
Theorem 2.7. For all affine transformations $\mathbf{J} \in G A(n, \mathbf{R})$ and projective transformations $\Phi \in G \mathbf{P}(n, \mathbf{R})$ the hyperplane mapping $\eta_{H}$ satisfies

$$
\eta_{H}(\mathbf{J} \circ \Phi)=\eta_{H}(\Phi) \text {. }
$$

The quotient mapping $\bar{\eta}_{H}: G A(n, \mathbf{R}) \backslash G \mathbf{P}(n, \mathbf{R}) \rightarrow \mathbf{P} H^{n}$ defined on the left coset space $G A(n, \mathbf{R}) \backslash G \mathbf{P}(n, \mathbf{R})$ is one-to-one and onto.

Proof. The equality (2.24) is immediate since affine transformations leave the hyperplane at infinity fixed. This shows that $\bar{\eta}_{H}$ is a well-defined mapping.

To see that $\bar{\eta}_{H}$ is one-to-one, suppose that $\Phi_{1}$ and $\Phi_{2}$ map the same hyperplane to the hyperplane at infinity. Then $\Phi_{2}^{-1} \circ \Phi_{1}$ maps the hyperplane at infinity to itself, hence is an affine transformation, so $\Phi_{2}=\mathbf{J} \circ \Phi_{1}$ and $\left[\Phi_{1}\right]=\left[\Phi_{2}\right]$. Since the group $G \mathbf{P}(n, \mathbf{R})$ has a transitive action on hyperplanes, the mapping is onto.

In the sequel we use a coordinatized version of the hyperplane mapping $\bar{\eta}_{H}$ in which $\mathbf{P} H^{n}$ is identified with $\left(\mathbf{P}^{n}\right)^{*}$ using the polarity correspondence (2.10). We denote the resulting map $\bar{\eta}_{P H}: G A(n, \mathbf{R}) \backslash G \mathbf{P}(n, \mathbf{R}) \rightarrow\left(\mathbf{P}^{n}\right)^{*}$ and call it the polar hyperplane mapping. Theorem 2.7 implies that it is one-to-one and onto. If the projective transformation $\Phi$ with domain $\mathbf{R}^{n}$ is

$$
\Phi\left(\left(x_{1}, \ldots, x_{n}\right)\right)=\left(\frac{a_{10}+\left\langle\mathbf{a}_{1}, \mathbf{x}\right\rangle}{a_{00}+\left\langle\mathbf{a}_{0}, \mathbf{x}\right\rangle}, \ldots, \frac{a_{n 0}+\left\langle\mathbf{a}_{n}, \mathbf{x}\right\rangle}{a_{00}+\left\langle\mathbf{a}_{0}, \mathbf{x}\right\rangle}\right)
$$

then $\bar{\eta}_{P H}(\Phi)$ is in the affine dual space $\left(\mathbf{R}^{n}\right)^{*}$ if and only if $a_{00} \neq 0$, and in that case

$$
\bar{\eta}_{P H}([\Phi])=-\mathbf{a}_{0} / a_{00} .
$$

\section{Projective centering and the projective \\ LEGENDRE TRANSFORM COORDINATE MAPPING}

Karmarkar $[\mathrm{K}]$ defined a notion of center of a set of linear program constraints $\mathrm{H}$ of a special form called canonical form, and proved that for any feasible point $\mathbf{x}$ there exists a suitable projective transformation $\Phi$ of a special form such that $\Phi(\mathbf{x})$ is the center of the transformed set of constraints $\Phi(\mathrm{H})$. In problems in Karmarkar's canonical form the polytope $P_{\mathrm{H}}$ of feasible solutions is always of lower dimension than the space it inhabits. In this section we derive analogues of Karmarkar's concepts valid for linear program constraint sets in inequality form which have a bounded full-dimensional polytope of feasible solutions. We introduce appropriate notions of center and of admissible projective transformation valid for such linear programs. The notion of center we use coincides with the "analytical center" of Sonnevend [So1, So2].

We consider a set $\mathrm{H}$ of linear program constraints in $\mathbf{R}^{n}$ in the inequality form:

$$
\left\langle\mathbf{a}_{j}, \mathbf{x}\right\rangle \geq b_{j} ; \quad 1 \leq j \leq m
$$


Throughout this section we assume that the polytope $P_{\mathrm{H}}$ of feasible solutions is full-dimensional and bounded. The full-dimensional hypothesis is equivalent to the assertion that

$$
\operatorname{Int}\left(P_{\mathrm{H}}\right)=\left\{\mathbf{x}:\left\langle\mathbf{a}_{j}, \mathbf{x}\right\rangle>b_{j} \text { for } 1 \leq j \leq m\right\}
$$

is nonempty.

The center $\mathbf{x}_{\mathrm{H}}$ of $P_{\mathrm{H}}$ is that point $\mathbf{x}_{\mathrm{H}}$ which minimizes

$$
f_{\mathrm{H}}(\mathbf{x})=-\sum_{j=1}^{m} \log \left(\left\langle\mathbf{a}_{j}, \mathbf{x}\right\rangle-b_{j}\right)
$$

on $\operatorname{Int}\left(P_{\mathrm{H}}\right)$, or equivalently, which maximizes

$$
\exp \left(-f_{\mathbf{H}}(\mathbf{x})\right)=\prod_{j=1}^{m}\left(\left\langle\mathbf{a}_{j}, \mathbf{x}\right\rangle-b_{j}\right)
$$

on this region. It is relatively easy to show that $f_{\mathrm{H}}(\mathbf{x})$ is a concave function so that $\mathbf{x}_{\mathrm{H}}$ is unique if it exists, and that $\mathbf{x}_{\mathrm{H}}$ exists whenever $P_{\mathrm{H}}$ is bounded. We define the Legendre transform coordinate mapping $\phi_{\mathrm{H}}: \operatorname{Int}\left(P_{\mathrm{H}}\right) \rightarrow\left(\mathbf{R}^{n}\right)^{*}$ by

$$
\phi_{\mathbf{H}}(\mathbf{x})=\nabla f_{\mathbf{H}}(\mathbf{x})=-\sum_{j=1}^{m} \frac{\mathbf{a}_{j}}{\left\langle\mathbf{a}_{j}, \mathbf{x}\right\rangle-b_{j}} .
$$

The center $\mathbf{x}_{\mathrm{H}}$ is alternatively characterized as the unique point in $\operatorname{Int}\left(P_{\mathrm{H}}\right)$ such that

$$
\phi_{\mathrm{H}}\left(\mathbf{x}_{\mathrm{H}}\right)=\mathbf{0} \text {. }
$$

Part II shows that the center $\mathbf{x}_{H}$ is an affine invariant in the sense that if $\mathbf{J}: \mathbf{R}^{n} \rightarrow \mathbf{R}^{n}$ is an invertible affine transformation then

$$
\mathbf{x}_{\mathbf{J}(\mathrm{H})}=\mathbf{J}\left(\mathbf{x}_{\mathrm{H}}\right) \text {. }
$$

Here $\mathbf{J}(\mathbf{H})$ is the set of constraints

$$
\left\langle\mathbf{a}_{j}, \mathbf{J}^{-1}(\mathbf{y})\right\rangle \geq b_{j} ; \quad 1 \leq j \leq m .
$$

The center $\mathbf{x}_{\mathrm{H}}$ depends on the constraint set $\mathrm{H}$; one can find constraint sets $\mathrm{H}$ and $\mathrm{H}^{\prime}$ with $P_{\mathrm{H}}=P_{\mathrm{H}^{\prime}}$ but $\mathbf{x}_{\mathrm{H}} \neq \mathbf{x}_{\mathrm{H}^{\prime}}$.

We now study the effect that projective transformations have on moving the center. The concept of center is only defined for bounded polytopes $P$, so we only allow projective transformations $\Phi$ such that $\Phi(P)$ is a bounded polytope. We call such projective transformations (strictly) admissible for $P$, and denote the set of strictly admissible projective transformations for $P$ by $\operatorname{Admiss}(P)$. (Note that $\operatorname{Admiss}(P)$ depends only on the polytope $P$ and not on the constraints $H$ determining the polytope.) A projective transformation $\Phi$ is in $\operatorname{Admiss}(P)$ if and only if $H_{\Phi} \cap P=\varnothing$ where $H_{\Phi}$ is the hyperplane mapped to infinity by $\Phi$. In $\S 2 \mathrm{E}$ we showed that the hyperplane $H_{\Phi}$ is unaffected by a subsequent affine transformation, and that the hyperplane mapping 
$\eta_{H}: G A(n, \mathbf{R}) \backslash G \mathbf{P}(n, \mathbf{R}) \rightarrow \mathbf{P} H^{n}$ with $\eta_{H}([\Phi])=H_{\Phi}$ is one-to-one and onto. Consequently we infer that for any bounded polytope $P$ the set $\operatorname{Admiss}(P)$ is a union of left cosets in $G A(n, \mathbf{R}) \backslash G \mathbf{P}(n, \mathbf{R})$.

Given a set of constraints $\mathrm{H}$, we say that an admissible projective transformation $\Phi$ for $P_{\mathrm{H}}$ centers $\mathbf{x}$ for $\mathrm{H}$ if $\mathbf{x} \in \operatorname{Int}\left(P_{\mathrm{H}}\right)$ and $\Phi(\mathbf{x})$ is the center of the set of constraints $\Phi(\mathrm{H})$.

Theorem 3.1. Let $\mathrm{H}$ be a set of linear inequality constraints in $\mathbf{R}^{n}$ having a bounded full-dimensional polytope of feasible solutions $P_{\mathrm{H}}$. For each $\mathbf{x} \in \operatorname{Int}\left(P_{\mathrm{H}}\right)$ there exists a projective transformation $\Phi \in \operatorname{Admiss}\left(P_{\mathbf{H}}\right)$ that centers $\mathbf{x}$. The set of all projective transformations that center $\mathbf{x}$ is exactly the left coset

$$
[\Phi]=\{\mathbf{J} \circ \Phi: J \in G A(n, \mathbf{R})\}
$$

in $G A(n, \mathbf{R}) \backslash G \mathbf{P}(n, \mathbf{R})$.

This theorem provides a well-defined mapping

$$
\mu_{\mathrm{H}}: \operatorname{Int}\left(P_{\mathrm{H}}\right) \rightarrow G A(n, \mathbf{R}) \backslash G \mathbf{P}(n, \mathbf{R})
$$

which assigns to each point $\mathbf{x} \in \operatorname{Int}\left(P_{\mathrm{H}}\right)$ the coset $[\Phi]$ in $G A(n, \mathbf{R}) \backslash G \mathbf{P}(n, \mathbf{R})$ of projective transformations that center $\mathbf{x}$. The theorem asserts that this mapping is one-to-one. Its range is $G A(n, \mathbf{R}) \backslash \operatorname{Admiss}\left(P_{\mathrm{H}}\right)$, because every transformation in $\operatorname{Admiss}\left(P_{\mathrm{H}}\right)$ gives an arrangement $\Phi(H)$ having a center $\mathbf{x}_{\Phi(H)}$ and so centers $\Phi^{-1}\left(\mathbf{x}_{\Phi(H)}\right)$. By composing this with the polar hyperplane mapping $\bar{\eta}_{H}$ we obtain a mapping.

$$
\Psi_{\mathrm{H}}=\bar{\eta}_{H} \circ \mu_{\mathrm{H}}: \operatorname{Int}\left(P_{\mathrm{H}}\right) \rightarrow \mathbf{P} H^{n},
$$

which we define to be the (abstract) projective Legendre transform coordinate mapping. We will later show this mapping "linearizes" $P$-trajectories. For explicit computations we use the related mapping obtained by identifying the space of hyperplanes of $\mathbf{P} H^{n}$ with $\left(\mathbf{P}^{n}\right)^{*}$ using the polarity correspondence and call this the projective Legendre transform coordinate mapping. It is

$$
\psi_{\mathrm{H}}=\bar{\eta}_{P H} \circ \mu_{\mathrm{H}}: \operatorname{Int}\left(P_{\mathrm{H}}\right) \rightarrow\left(\mathbf{P}^{n}\right)^{*},
$$

where $\eta_{P H}$ is the polar hyperplane mapping defined in $\S 2 \mathrm{E}$.

Proof of Theorem 3.1. Let $\mathbf{x}_{0} \in \operatorname{Int}\left(P_{\mathrm{H}}\right)$ be given. Using the translation $\mathbf{T}(\mathbf{x})=$ $\mathbf{x}-\mathbf{x}_{0}$ we easily see that

$$
\Phi \text { centers } \mathbf{x}_{0} \text { for } H \Leftrightarrow \Phi \circ \mathbf{T}^{-1} \text { centers } \mathbf{0} \text { for } \mathbf{T}(H) \text {. }
$$

Thus it suffices to prove the theorem in the special case $\mathbf{x}_{0}=\mathbf{0}$. This is accomplished by the following lemma.

Lemma 3.1. Let $\mathrm{H}$ be a set of inequality constraints having a bounded polytope $P_{\mathrm{H}}$ of feasible solutions in $\mathbf{R}^{n}$ which contains $\mathbf{0}$ in its interior. Then there exists a unique vector $\mathbf{p}$ in $\left(\mathbf{R}^{n}\right)^{*}$ such that the projective transformation

$$
\Phi_{\mathbf{p}}(\mathbf{x})=\frac{\mathbf{x}}{1-\langle\mathbf{p}, \mathbf{x}\rangle}
$$


is in $\operatorname{Admiss}\left(P_{\mathrm{H}}\right)$ and centers $\mathbf{0}$ for $\mathrm{H}$. The vector $-\mathbf{p}$ is in the interior of the polar polytope $P_{\mathrm{H}}^{\circ}$.

We defer the proof of the lemma and complete the proof of Theorem 3.1 assuming it is proved. The lemma proves the existence of a projective transformation $\Phi_{p}$ of the special form (3.9) that centers 0 for $H$. Since the center is an affine invariant every projective transformation in $\left[\Phi_{\mathbf{p}}\right]=\left\{\mathbf{J} \circ \Phi_{\mathbf{p}}: \mathbf{J} \in\right.$ $G A(n, \mathbf{R})\}$ centers 0 for $\mathrm{H}$. Now suppose that $\Phi_{1}$ centers 0 for $\mathrm{H}$. Set

$$
\Phi_{2}=\mathbf{D} \circ \mathbf{T} \circ \Phi_{1},
$$

where $\mathbf{T}$ is the translation $\mathbf{T}(\mathbf{y})=\mathbf{y}-\Phi_{1}(\mathbf{0})$, and $\mathbf{D}$ the linear transformation

$$
\mathbf{D}(\mathbf{y})=\left[\nabla \Phi_{1}(\mathbf{0})\right]^{-1} \mathbf{y},
$$

where

$$
\nabla \Phi_{1}(\mathbf{x})=\left[\frac{\partial}{\partial x_{i}} \Phi_{1}(\mathbf{x})_{j}\right],
$$

denotes the Jacobian of $\Phi_{1}$ at $\mathbf{x}$. Then $\Phi_{2}(\mathbf{0})=\mathbf{0}$ and $\nabla \Phi_{2}(\mathbf{0})=I$, which forces the projective transformation $\Phi_{2}$ to have the form

$$
\Phi_{2}(\mathbf{x})=\frac{\mathbf{x}}{1-\langle\mathbf{q}, \mathbf{x}\rangle}
$$

for some $\mathbf{q}$. The uniqueness assertion of Lemma 3.1 then implies that $\Phi_{2} \equiv \Phi_{p}$ and (3.10) then shows $\Phi_{1} \in\left[\Phi_{p}\right]$, which completes the proof of Theorem 3.1.

It remains to prove Lemma 3.1. To do this, we first show that a projective transformation $\Phi_{\mathbf{p}} \in \operatorname{Admiss}\left(P_{\mathrm{H}}\right)$ if and only if $\mathbf{p} \in \operatorname{Int}\left(P_{\mathrm{H}}^{\circ}\right)$. Indeed we have $H_{\Phi}=\{\mathbf{x}: 1-\langle\mathbf{p}, \mathbf{x}\rangle=0\}$ and the condition that $H_{\Phi} \cap P_{\mathrm{H}}=\varnothing$ means that $1-\langle\mathbf{p}, \mathbf{x}\rangle$ has a constant sign for all $\mathbf{x} \in P_{\mathrm{H}}$. Since $\mathbf{0} \in P_{\mathrm{H}}$ this sign is positive, so that $\langle\mathbf{p}, \mathbf{x}\rangle<1$ for all $\mathbf{x} \in P_{\mathrm{H}}$, which says that $\mathbf{p} \in \operatorname{Int}\left(P_{\mathrm{H}}^{\circ}\right)$. Conversely, if $\mathbf{p} \in \operatorname{Int}\left(P_{\mathrm{H}}\right)$ the reverse argument shows that $H_{\Phi} \cap P_{\mathrm{H}}=\varnothing$.

Now suppose $\mathrm{H}$ has the form $\left\langle\mathbf{a}_{j}, \mathbf{x}\right\rangle \geq b_{j} ; 1 \leq j \leq m$. The condition $\mathbf{0} \in \operatorname{Int}\left(P_{\mathbf{H}}\right)$ implies that

$$
b_{j}<0 ; \quad 1 \leq j \leq m .
$$

If $\mathbf{y}=\Phi_{\mathbf{p}}(\mathbf{x})$ then

$$
\mathbf{x}=\Phi_{\mathbf{p}}^{-1}(\mathbf{y})=\Phi_{-\mathbf{p}}(\mathbf{y})=\frac{\mathbf{y}}{1+\langle\mathbf{p}, \mathbf{y}\rangle}
$$

The constraints of $\Phi_{\mathbf{p}}(H)$ are then computed to be

$$
\left\langle\mathbf{a}_{j}, \Phi_{\mathbf{p}}^{-1}(\mathbf{y})\right\rangle \geq b_{j} ; \quad 1 \leq j \leq m,
$$

which are

$$
\left\langle\mathbf{a}_{j}-b_{j} \mathbf{p}, \mathbf{y}\right\rangle \geq b_{j} ; \quad 1 \leq j \leq m .
$$


We have $\Phi_{\mathbf{p}}(\mathbf{0})=\mathbf{0}$, and we use the fact that for $\Phi_{\mathbf{p}} \in \operatorname{Admiss}\left(P_{\mathrm{H}}\right)$ the condition that $\mathbf{0}$ is the center of $\tilde{H}=\Phi_{\mathbf{p}}(\mathbf{H})$ is that $\phi_{\tilde{H}}(\mathbf{0})=\mathbf{0}$, where

$$
\phi_{\hat{\mathrm{H}}}(\mathbf{y})=-\sum_{j=1}^{m} \frac{\mathbf{a}_{j}-b_{j} \mathbf{p}}{\left\langle\mathbf{a}_{j}-b_{j} \mathbf{p}, \mathbf{y}\right\rangle-b_{j}}
$$

is the Legendre transform coordinate mapping for $\tilde{H}$. Now (3.13) gives

$$
\phi_{\tilde{\mathrm{H}}}(\mathbf{0})=-m \mathbf{p}+\sum_{j=1}^{m} \frac{1}{b_{j}} \mathbf{a}_{j} \cdot
$$

The condition (3.11) guarantees that all $b_{j}$ are invertible. This equation shows that there is at most one vector $\mathbf{p}$ making $\phi_{\tilde{\mathbf{H}}}(\mathbf{0})=\mathbf{0}$, which is

$$
\mathbf{p}=\frac{1}{m} \sum_{j=1}^{m} \frac{1}{b_{j}} \mathbf{a}_{j}
$$

To prove existence we must check that $\Phi_{\mathbf{p}} \in \operatorname{Admiss}\left(P_{\mathrm{H}}\right)$ for $\mathbf{p}$ satisfying (3.15). We first show that

$$
1-\langle\mathbf{p}, \mathbf{x}\rangle \geq 0, \quad \text { all } \mathbf{x} \in P_{\mathrm{H}} .
$$

Indeed

$$
1-\langle\mathbf{p}, \mathbf{x}\rangle=1-\frac{1}{m} \sum_{j=1}^{m} \frac{1}{b_{j}}\left\langle\mathbf{a}_{j}, \mathbf{x}\right\rangle .
$$

Now if $\mathbf{x} \in P_{\mathrm{H}}$ then $\left\langle\mathbf{a}_{j}, \mathbf{x}\right\rangle \geq b_{j}$ so the last equality implies (3.16) holds. Next we claim that

$$
1-\langle\mathbf{p}, \mathbf{x}\rangle>0 \text { for all } \mathbf{x} \in P_{\mathrm{H}},
$$

i.e. at least one $\left\langle\mathbf{a}_{j}, \mathbf{x}\right\rangle>b_{j}$ holds if $\mathbf{x} \in P_{\mathbf{H}}$. Suppose not. Then $\operatorname{since} \operatorname{Int}\left(P_{\mathrm{H}}\right)$ is nonempty, $P_{\mathrm{H}}$ contains two points $\mathbf{x}_{0}$ and $\mathbf{x}_{1}$, with all $\left\langle\mathbf{a}_{j}, \mathbf{x}_{i}\right\rangle=b_{j}$ for $i=1,2$, so that $P_{\mathrm{H}}$ contains the unbounded ray $\left\{\mathbf{x}_{0}+\lambda\left(\mathbf{x}_{1}-\mathbf{x}_{0}\right): \lambda \geq 0\right\}$, a contradiction. This proves (3.17) holds, so $\Phi_{\mathbf{p}}$ is admissible for $\mathbf{p}$. This proves the existence of $\mathbf{p}$, and it is unique by (3.15). Lemma 3.1 is proved.

The proof of Theorem 3.1 gives an algorithm which when given a set of constraints $\mathrm{H}$ and a point $\mathbf{x} \in \operatorname{Int}\left(P_{\mathrm{H}}\right)$ finds a projective transformation $\Phi$ that centers $\mathbf{x}$ for $H$. Simply translate $\mathbf{x}$ to $\mathbf{0}$ and find $\Phi_{\mathbf{p}}$ of Lemma 3.1 using the formula (3.15).

We use this procedure to compute an explicit formula for the projective Legendre transform coordinate mapping $\psi_{\mathrm{H}}$.

Theorem 3.2. Let $\mathrm{H}$ be the set of constraints

$$
\left\langle\mathbf{a}_{j}, \mathbf{x}\right\rangle \geq b_{j} ; \quad 1 \leq j \leq m,
$$

such that its polytope $P_{\mathrm{H}}$ of feasible solutions in $\mathbf{R}^{n}$ is bounded with $\mathbf{0} \in \operatorname{Int}\left(P_{\mathrm{H}}\right)$. The projective Legendre transform mapping $\psi_{\mathrm{H}}$ : Int $P_{\mathrm{H}} \rightarrow\left(\mathbf{P}^{n}\right)^{*}$ is given by

$$
\psi_{\mathrm{H}}(\mathbf{x})=\frac{\phi_{\mathrm{H}}(\mathbf{x})}{m+\left\langle\phi_{\mathrm{H}}(\mathbf{x}), \mathbf{x}\right\rangle}
$$


where

$$
\phi_{\mathrm{H}}(\mathbf{x})=-\sum_{j=1}^{m} \frac{\mathbf{a}_{j}}{\left\langle\mathbf{a}_{j}, \mathbf{x}\right\rangle-b_{j}}
$$

is the Legendre transform coordinate mapping. The mapping $\psi_{\mathrm{H}}$ is a one-to-one mapping with range $\operatorname{Int}\left(P_{\mathrm{H}}^{\circ}\right)$ where $P_{\mathrm{H}}^{\circ}$ is the polytope polar to $P_{\mathrm{H}}$.

Proof. Suppose $\mathbf{x}_{0} \in \operatorname{Int}\left(P_{H}\right)$ is given, and we wish to compute $\psi_{H}\left(\mathbf{x}_{0}\right)$. First, translate $\mathbf{x}_{0}$ to the origin $\mathbf{0}$ using $\mathbf{y}=\mathbf{T}(\mathbf{x})=\mathbf{x}-\mathbf{x}_{0}$. The new constraints $\tilde{H}=\mathbf{T}(H)$ are given by

$$
\left\langle\mathbf{a}_{j}, \mathbf{T}^{-1}(\mathbf{y})\right\rangle \geq b_{j} ; \quad 1 \leq j \leq m,
$$

which are

$$
\left\langle\mathbf{a}_{j}, \mathbf{y}\right\rangle \geq b_{j}-\left\langle\mathbf{a}_{j}, \mathbf{x}_{0}\right\rangle .
$$

The proof of Lemma 3.1 showed that the point $\mathbf{0}$ is centered for $\tilde{H}$ by

$$
\Phi_{\mathbf{p}}(\mathbf{y})=\frac{\mathbf{y}}{1-\langle\mathbf{p}, \mathbf{y}\rangle}
$$

with

$$
\mathbf{p}=\frac{1}{m} \sum_{j=1}^{m} \frac{1}{b_{j}-\left\langle\mathbf{a}_{j}, \mathbf{x}_{0}\right\rangle} \mathbf{a}_{j}
$$

using (3.15). Note that

$$
\mathbf{p}=\frac{1}{m} \phi_{H}\left(\mathbf{x}_{0}\right)
$$

where $\phi_{H}(\mathbf{x})$ is the Legendre transform coordinate map (3.19). Now $\Phi=\Phi_{\mathbf{p}} \circ \mathbf{T}$ centers $\mathbf{x}_{0}$ for $H$, and

$$
\Phi(\mathbf{x})=\frac{\mathbf{x}-\mathbf{x}_{0}}{1-\left\langle\mathbf{p}, \mathbf{x}-\mathbf{x}_{0}\right\rangle}
$$

We have

$$
H_{\Phi}=\left\{\mathbf{x}: 1-\left\langle\mathbf{p}, \mathbf{x}-\mathbf{x}_{0}\right\rangle=0\right\},
$$

and using (3.21) we find that

$$
H_{\Phi}=\left\{\mathbf{x}: 1-\frac{\frac{1}{m}\left\langle\phi_{H}\left(\mathbf{x}_{0}\right), \mathbf{x}\right\rangle}{1+\frac{1}{m}\left\langle\phi_{H}\left(\mathbf{x}_{0}\right), \mathbf{x}_{0}\right\rangle}=0\right\} .
$$

By definition of the polarity correspondence the projective Legendre transform mapping $\psi_{\mathrm{H}}\left(\mathbf{x}_{0}\right)$ satisfies

$$
H_{\Phi}=\left\{\mathbf{x}: 1-\left\langle\psi_{H}\left(\mathbf{x}_{0}\right), \mathbf{x}\right\rangle=0\right\},
$$

so that

which is $(3.18)$.

$$
\psi_{H}(\mathbf{x})=\frac{\phi_{H}\left(\mathbf{x}_{0}\right)}{m+\left\langle\phi_{H}\left(\mathbf{x}_{0}\right), \mathbf{x}_{0}\right\rangle}
$$


Theorem 3.1 implies that the projective Legendre transform mapping $\psi_{\mathrm{H}}$ is a one-to-one mapping onto its range. This range is contained in $\operatorname{Int}\left(P_{\mathrm{H}}^{\circ}\right)$ because under the polarity mapping it corresponds to hyperplanes $H$ with $H \cap P_{\mathrm{H}}=\varnothing$, and all admissible projective transformations $\Phi$ have $H_{\Phi}$ with this property. The range contains $\operatorname{Int}\left(P_{\mathrm{H}}^{\circ}\right)$ because every hyperplane $H$ with $H \cap P_{\mathrm{H}} \neq \varnothing$ is the hyperplane sent to infinity for some projective transformation $\Phi$, and since $\Phi\left(P_{H}^{\circ}\right)$ is bounded it has a center $\mathbf{x}_{\Phi(H)}$ and $\Phi$ centers $\Phi^{-1}\left(\mathbf{x}_{\Phi(H)}\right)$ for $\mathrm{H}$. Hence the range is $\operatorname{Int}\left(P_{\mathrm{H}}\right)$.

It is possible to extend the results of this section to all sets of linear program constraints having a bounded polytope of feasible solutions of arbitrary codimension in the coordinate space. The definition of center used in this case is the one in part II. A projective transformation $\Phi$ is admissible if it is invertible on the flat $M P_{\mathrm{H}}$ determined by $P_{\mathrm{H}}$ and if $H_{\Phi} \cap P_{\mathrm{H}}=\varnothing$. Let $D_{\mathrm{H}}$ denote the feasible direction subspace for $\mathrm{H}$, which is the subspace of $\mathbf{R}^{n}$ parallel to $M P_{\mathrm{H}}$. The appropriate image space of the projective Legendre transformation mapping $\psi_{\mathrm{H}}$ in this case is the projective completion of the affine space $\left(\mathbf{R}^{n}\right)^{*} / D_{\mathrm{H}}^{P}$. We omit further details.

\section{ADJOINT PROJECTIVE TRANSFORMATIONS AND} THE PROJECTIVE LEGENDRE TRANSFORM COORDINATE MAPPING

This section shows that the projective Legendre transform mappings are compatible with projective transformations $\Phi$ and polar adjoint transformations $\Phi^{P A}$

Theorem 4.1. Let $\mathrm{H}$ be a set of constraints having a bounded nonempty polytope $P_{\mathrm{H}}$ of feasible solutions in $\mathbf{R}^{n}$ such that $\mathbf{0}$ is in $\operatorname{Int}\left(P_{\mathrm{H}}\right)$. Let $\Phi \in \operatorname{Admiss}\left(P_{\mathrm{H}}\right)$ be such that $\mathbf{0}$ is in $\operatorname{Int}\left(P_{\Phi(\mathrm{H})}\right)$. Then all maps in the following diagram are one-to-one and onto and the diagram commutes.

$$
\begin{array}{ccc}
\operatorname{Int}\left(P_{\mathrm{H}}\right) & \stackrel{\Phi}{\longrightarrow} & \operatorname{Int}\left(P_{\Phi(\mathrm{H})}\right) \\
\psi_{\mathrm{H}} \downarrow & & \downarrow \psi_{\Phi(\mathrm{H})} \\
\operatorname{Int}\left(P_{\mathrm{H}}^{\circ}\right) & \stackrel{\Phi^{P A}}{ } & \operatorname{Int}\left(P_{\Phi(\mathrm{H})}^{\circ}\right)
\end{array}
$$

Here $\psi_{\mathrm{H}}$ and $\psi_{\Phi(\mathrm{H})}$ are projective Legendre transform maps and $\Phi^{P A}$ is the polar adjoint map to $\Phi$.

Theorem 4.1 can be viewed as an extension of Klee's adjoint projective mapping correspondence. The hypotheses that $0 \in \operatorname{Int}\left(P_{H}\right)$ and $0 \in \operatorname{Int}\left(P_{\Phi(H)}\right)$ seem restrictive but are not; they arise from the use of the polarity correspondence. It is possible to prove a coordinate-free version of this theorem valid for all admissible projective transformations, which yields

$$
\begin{array}{ccc}
\operatorname{Int}\left(P_{\mathrm{H}}\right) & \stackrel{\Phi}{\longrightarrow} & \operatorname{Int}\left(P_{\Phi(\mathrm{H})}\right) \\
\hat{\psi}_{\mathrm{H}} \downarrow & & \downarrow \hat{\psi}_{\Phi(\mathrm{H})} \\
\operatorname{Int}\left(P_{\mathrm{H}}^{d}\right) & \stackrel{\Phi^{A}}{\longleftarrow} & \operatorname{Int}\left(P_{\Phi(\mathrm{H})}^{d}\right)
\end{array}
$$


Proof of Theorem 4.1. Under the hypotheses the mappings $\psi_{\mathrm{H}}, \psi_{\Phi(\mathrm{H})}$ are oneto-one and onto by Theorem 3.2, while $\Phi$ and $\Phi^{P A}$ are one-to-one and onto by Theorem 2.6. So we need only verify the commutativity property $(4.1)$ which is:

$$
\psi_{\mathrm{H}}(\mathbf{x})=\Phi^{P A} \circ \psi_{\Phi(\mathrm{H})} \circ \Phi(\mathbf{x}), \quad \mathbf{x} \in \operatorname{Int}\left(P_{\mathrm{H}}\right) .
$$

We first reduce this problem to simpler special cases. Any projective transformation $\Phi$ which does not send 0 to infinity has a representation of the hyperplane $H_{\Phi}$ it sends to infinity as

$$
H_{\Phi}=\{\mathbf{x}: 1-\langle\mathbf{p}, \mathbf{x}\rangle=0\}
$$

for a unique $\mathbf{p}$. Any such $\Phi$ has a decomposition $\Phi=\Phi_{3} \circ \Phi_{2} \circ \Phi_{1}$ where

$$
\Phi_{1}(\mathbf{x})=\frac{\mathbf{x}}{1-\langle\mathbf{p}, \mathbf{x}\rangle} \quad \text { and } \quad \Phi_{2}(\mathbf{x})=A \mathbf{x}
$$

is an invertible linear transformation with $A=\nabla \Phi(\mathbf{0})$, and $\Phi_{3}(\mathbf{x})=\mathbf{x}+\mathbf{x}_{0}$ is a translation with $\mathbf{x}_{0}=\Phi(\mathbf{0})$. (This decomposition is unique.)

It suffices to prove the theorem for $\Phi_{1}, \Phi_{3}$ and $\Phi_{3}$ separately, because if the hypotheses of the theorem hold for $(\Phi, \mathbf{H})$ then they also hold for $\left(\Phi_{1}, H\right)$, $\left(\Phi_{2}, \Phi_{1}(\mathrm{H})\right)$ and $\left(\Phi_{3}, \Phi_{2} \circ \Phi_{1}(\mathrm{H})\right)$. To see this, suppose that $\Phi \in \operatorname{Admiss}\left(P_{\mathrm{H}}\right)$ and $\mathbf{0} \in \operatorname{Int}\left(P_{\mathbf{H}}\right)$. Since $\Phi_{2}, \Phi_{3}$ are affine, $[\Phi]=\left[\Phi_{1}\right]$ in $G A(n, \mathbf{R}) \backslash G \mathbf{P}(n, \mathbf{R})$ so that $\Phi_{1} \in \operatorname{Admiss}\left(P_{\mathrm{H}}\right)$ and $P_{\Phi_{1}(\mathrm{H})}$ is bounded. Then since $\Phi_{2}, \Phi_{3}$ are affine they are admissible for any bounded polytope, and $\Phi_{2} \circ \Phi_{1}(H)$ is bounded. Finally since $\Phi_{1}(\mathbf{0})=\mathbf{0}, \Phi_{2}(\mathbf{0})=\mathbf{0}$ the hypotheses that $\mathbf{0} \in \operatorname{Int}\left(P_{\Phi_{1}(\mathrm{H})}\right)$ and $\mathbf{0} \in \operatorname{Int}\left(P_{\Phi_{2} \circ \Phi_{1}(\mathrm{H})}\right)$ both hold.

We suppose that the constraints $\mathrm{H}$ are $\left\langle\mathbf{a}_{j}, \mathbf{x}_{j}\right\rangle \geq b_{j}, 1 \leq j \leq m$.

Case 1. $\Phi(\mathbf{x})=\mathbf{x} /(1-\langle\mathbf{p}, \mathbf{x}\rangle)$. In this case, using (2.19) we have

$$
\Phi^{P A}(\mathbf{u})=\mathbf{u}+\mathbf{p} \text {. }
$$

Let $\mathbf{w} \in \operatorname{Int}\left(P_{\mathrm{H}}\right)$ be given. Then

$$
\hat{\mathbf{w}} \equiv \psi_{\mathrm{H}}(\mathbf{w})=\frac{\phi_{\mathrm{H}}(\mathbf{w})}{m+\left\langle\phi_{\mathbf{H}}(\mathbf{w}), \mathbf{w}\right\rangle}
$$

where $\phi_{H}(\cdot)$ is the Legendre transform coordinate map for $H$, i.e.

$$
\phi_{\mathbf{H}}(\mathbf{w})=-\sum_{j=1}^{m} \frac{\mathbf{a}_{j}}{\left\langle\mathbf{a}_{j}, \mathbf{w}\right\rangle-b_{j}} .
$$

We begin computing $\Phi^{P A}=\psi_{\Phi(\mathrm{H})} \circ \Phi(\mathbf{w})$ with

$$
\mathbf{y}=\Phi(\mathbf{w})=\frac{\mathbf{w}}{1-\langle\mathbf{p}, \mathbf{w}\rangle} \text {. }
$$

Next, letting $\tilde{H}=\Phi(H)$, we have

$$
\hat{\mathbf{y}} \equiv \psi_{\tilde{\mathrm{H}}}(\mathbf{y})=\frac{\phi_{\tilde{\mathrm{H}}}(\mathbf{y})}{m+\left\langle\phi_{\tilde{\mathrm{H}}}(\mathbf{y}), \mathbf{y}\right\rangle} .
$$


To compute $\phi_{\hat{H}}$ we note that the constraints $\Phi(H)$ are

$$
\left\langle\mathbf{a}_{j}-b_{j} \mathbf{p}, \mathbf{y}\right\rangle-b_{j} \geq 0 ; \quad 1 \leq j \leq m,
$$

as computed in Lemma 3.1, cf. (3.12). Hence

$$
\phi_{\tilde{\mathrm{H}}}(\mathbf{y})=-\sum_{j=1}^{m} \frac{\mathbf{a}_{j}-b_{j} \mathbf{p}}{\left\langle\mathbf{a}_{j}-b_{j} \mathbf{p}, \mathbf{y}\right\rangle-b_{j}} .
$$

Using (4.8) we have

$$
\begin{aligned}
m+\left\langle\phi_{\tilde{\mathrm{H}}}(\mathbf{y}), \mathbf{y}\right\rangle & =m-\sum_{j=1}^{m} \frac{\left\langle\mathbf{a}_{i}-b_{i} \mathbf{p}, \mathbf{y}\right\rangle}{\left\langle\mathbf{a}_{i}-b_{j} \mathbf{p}, \mathbf{y}\right\rangle-b_{j}} \\
& =-\sum_{j=1}^{m} \frac{b_{j}}{\left\langle\mathbf{a}_{j}-b_{j} \mathbf{p}, \mathbf{y}\right\rangle-b_{j}}
\end{aligned}
$$

Substituting the last two equations into (4.8) gives

$$
\hat{\mathbf{y}}=\frac{\sum_{j=1}^{m} \frac{\mathbf{a}_{j}-b_{j} \mathbf{p}}{\left\langle\mathbf{a}_{j}-b_{j} \mathbf{p}, \mathbf{y}\right\rangle-b_{j}}}{\sum_{j=1}^{m} \frac{b_{j}}{\left\langle\mathbf{a}_{j}-b_{j} \mathbf{p}, \mathbf{y}\right\rangle-b_{j}}} .
$$

Now using (4.4) we have

$$
\hat{\hat{\mathbf{w}}} \equiv \Phi^{P A} \circ \psi_{\Phi(\mathrm{H})} \circ \Phi(\mathbf{w})=\Phi^{P A}(\hat{\mathbf{y}})=\hat{\mathbf{y}}+\mathbf{p},
$$

and using (4.9) for $\hat{\mathbf{y}}$ gives

$$
\hat{\hat{\mathbf{w}}}=\frac{\sum_{j=1}^{m} \frac{\mathbf{a}_{j}}{\left\langle\mathbf{a}_{j}-b_{j} \mathbf{p}, \mathbf{y}\right\rangle-b_{j}}}{\sum_{j=1}^{m} \frac{b_{j}}{\left\langle a_{j}-b_{j} \mathbf{p}, \mathbf{y}\right\rangle-b_{j}}} .
$$

Now we substitute (4.7) into this expression and clear the denominator 1 $\langle\mathbf{p}, \mathbf{w}\rangle$ to get

$$
\hat{\hat{\mathbf{w}}}=\frac{\sum_{j=1}^{m} \frac{\mathbf{a}_{j}(1-\langle\mathbf{p}, \mathbf{w}\rangle)}{\left\langle\mathbf{a}_{j}-b_{j} \mathbf{p}, \mathbf{w}\right\rangle-b_{j}(1-\langle\mathbf{p}, \mathbf{w}\rangle)}}{\left.\sum_{j=1}^{m} \frac{b_{j}(1-\langle\mathbf{p}, \mathbf{w}\rangle)}{\left\langle\mathbf{a}_{j}-b_{j} \mathbf{p}, \mathbf{w}\right\rangle-b_{j}(1-\langle\mathbf{p}, \mathbf{w}\rangle}\right)}=\frac{\sum_{j=1}^{m} \frac{\mathbf{a}_{j}}{\left\langle\mathbf{a}_{j}, \mathbf{w}\right\rangle-b_{j}}}{\sum_{j=1}^{m} \frac{b_{j}}{\left\langle\mathbf{a}_{j}, \mathbf{w}\right\rangle-b_{j}}},
$$

after cancelling $1-\langle\mathbf{p}, \mathbf{w}\rangle$ from numerator and denominator. The numerator in (4.10) is $-\phi_{H}(\mathbf{w})$ by (4.6) and the denominator is

$$
\sum_{j=1}^{m} \frac{b_{j}}{\left\langle\mathbf{a}_{j}, \mathbf{w}\right\rangle-b_{j}}=-m+\sum_{j=1}^{m} \frac{\left\langle\mathbf{a}_{j}, \mathbf{w}\right\rangle}{\left\langle\mathbf{a}_{j}, \mathbf{w}\right\rangle-b_{j}}=-m-\left\langle\phi_{\mathrm{H}}(\mathbf{w}), \mathbf{w}\right\rangle .
$$

Hence

$$
\hat{\hat{\mathbf{w}}}=\frac{\phi_{\mathbf{H}}(\mathbf{w})}{m+\left\langle\phi_{\mathbf{H}}(\mathbf{w}), \mathbf{w}\right\rangle},
$$

which agrees with (4.5) and completes Case 1. 
Case 2. $\Phi(\mathbf{x})=A \mathbf{x}$. In this case (2.19) gives $\Phi^{P A}(\mathbf{u})=A^{T} \mathbf{u}$. Now given $\mathbf{w} \in \operatorname{Int}\left(P_{\mathrm{H}}\right)$ we have

$$
\hat{\mathbf{w}} \equiv \psi_{\mathrm{H}}(\mathbf{w})=\frac{\phi_{\mathrm{H}}(\mathbf{w})}{m+\left\langle\phi_{\mathrm{H}}(\mathbf{w}), \mathbf{w}\right\rangle}
$$

where

$$
\phi_{\mathrm{H}}(\mathbf{w})=-\sum_{j=1}^{m} \frac{\mathbf{a}_{j}}{\left\langle\mathbf{a}_{j}, \mathbf{w}\right\rangle-b_{j}} .
$$

If $\tilde{H}=\Phi(H)$ then the constraints of $\tilde{H}$ are easily calculated to be

$$
\left\langle\left(A^{-1}\right)^{T} \mathbf{a}_{j}, \mathbf{y}\right\rangle \geq b_{j}
$$

Hence

$$
\phi_{\tilde{\mathrm{H}}}(\mathbf{y})=-\sum_{j=1}^{m} \frac{\left(A^{-1}\right)^{T} \mathbf{a}_{j}}{\left\langle\left(A^{-1}\right)^{T} \mathbf{a}_{j}, \mathbf{y}\right\rangle-b_{j}} .
$$

Now taking $\mathbf{y}=\Phi(\mathbf{w})=A \mathbf{w}$ yields

$$
\phi_{\tilde{H}}(\mathbf{y})=\left(A^{-1}\right)^{T} \phi_{H}(\mathbf{w})
$$

by comparison with (4.12). Hence

$$
\begin{aligned}
m+\left\langle\phi_{\tilde{H}}(\mathbf{y}), \mathbf{y}\right\rangle & =m+\left\langle\left(A^{-1}\right)^{T} \phi_{\mathrm{H}}(\mathbf{w}), A \mathbf{w}\right\rangle \\
& =m+\left\langle\phi_{\mathrm{H}}(\mathbf{w}), \mathbf{w}\right\rangle .
\end{aligned}
$$

Thus

$$
\begin{aligned}
\hat{\hat{\mathbf{w}}} & \equiv \Phi^{P A} \circ \psi_{\tilde{\mathrm{H}}} \circ \Phi(\mathbf{w}) \\
& =A^{T}\left(\frac{\phi_{\tilde{\mathrm{H}}}(\mathbf{y})}{m+\left\langle\phi_{\tilde{\mathrm{H}}}(\mathbf{y}), \mathbf{y}\right\rangle}\right) \\
& =\frac{\phi_{\mathrm{H}}(\mathbf{w})}{m+\left\langle\phi_{\mathrm{H}}(\mathbf{w}), \mathbf{w}\right\rangle},
\end{aligned}
$$

after using (4.14) and (4.15). This agrees with (4.11) and finishes Case 2.

Case 3. $\Phi(\mathbf{x})=\mathbf{x}+\mathbf{p}$. In this case (2.19) gives

$$
\Phi^{P A}(\mathbf{u})=\frac{\mathbf{u}}{1-\langle\mathbf{u}, \mathbf{p}\rangle}
$$

By definition

$$
\hat{\mathbf{w}}=\psi_{\mathrm{H}}(\mathbf{w})=\frac{\phi_{\mathrm{H}}(\mathbf{w})}{m+\left\langle\phi_{\mathrm{H}}(\mathbf{w}), \mathbf{w}\right\rangle}
$$

The constraints $\tilde{H}=\Phi(H)$ are easily computed to be

$$
\left\langle\mathbf{a}_{j}, \mathbf{y}\right\rangle \geq b_{j}+\left\langle\mathbf{a}_{j}, \mathbf{p}\right\rangle ; \quad 1 \leq j \leq m,
$$


so that

$$
\phi_{\tilde{\mathbf{H}}}(\mathbf{y})=-\sum_{j=1}^{m} \frac{\mathbf{a}_{j}}{\left\langle\mathbf{a}_{j}, \mathbf{y}\right\rangle-\left(b_{j}+\left\langle\mathbf{a}_{j}, \mathbf{p}\right\rangle\right)} .
$$

Now choose $\mathbf{y}=\Phi(\mathbf{w})=\mathbf{w}+\mathbf{p}$ and the last equation gives

$$
\phi_{\tilde{\mathrm{H}}}(\mathbf{y})=\phi_{\mathrm{H}}(\mathbf{w}) \text {. }
$$

Then

$$
\hat{\mathbf{y}}=\psi_{\tilde{H}}(\mathbf{y})=\frac{\phi_{H}(\mathbf{w})}{m+\left\langle\phi_{H}(\mathbf{w}), \mathbf{w}+\mathbf{p}\right\rangle} .
$$

Hence

$$
\begin{aligned}
\hat{\hat{\mathbf{w}}} & =\Phi^{P A} \circ \psi_{\tilde{H}} \circ \Phi(\mathbf{w})=\Phi^{P A}(\hat{\mathbf{y}})=\frac{\hat{\mathbf{y}}}{1-\langle\hat{\mathbf{y}}, \mathbf{p}\rangle} \\
& =\frac{\cdot \phi_{H}(\mathbf{w})}{\left(m+\left\langle\phi_{H}(\mathbf{w}), \mathbf{w}+\mathbf{p}\right\rangle\right)-\left\langle\phi_{H}(\mathbf{w}), \mathbf{p}\right\rangle}=\frac{\phi_{H}(\mathbf{w})}{m+\left\langle\phi_{H}(\mathbf{w}), \mathbf{w}\right\rangle}
\end{aligned}
$$

which agrees with (4.16) and finishes Case 3 and the proof.

\section{Projective SCALING DIRECTION FIELD FOR FULL-DIMENSIONAL LINEAR PROGRAMS}

Karmarkar defined the projective scaling algorithm for linear programs of a special form we call canonical form. Canonical form linear programs always have a bounded polytope of feasible solutions and since they contain equality constraints, this polytope is not of full dimension. This section defines the projective scaling direction field for a linear program in $\mathbf{R}^{n}$ given in inequality form:

$$
\left\{\begin{array}{l}
\operatorname{minimize}\langle\mathbf{c}, \mathbf{x}\rangle-c_{0} \\
\left\langle\mathbf{a}_{j}, \mathbf{x}\right\rangle \geq b_{j} ; \quad 1 \leq j \leq m,
\end{array}\right.
$$

whose polytope $P_{\mathrm{H}}$ of feasible solutions is full-dimensional and bounded.

There are two equivalent ways to define the projective scaling direction field for such a problem. The first definition is to find an injective affine transformation $\mathbf{J}: \mathbf{R}^{n} \rightarrow \mathbf{R}^{m}$ that maps the inequality form linear program into a canonical form linear program in $\mathbf{R}^{m}$, and then to pull back the direction specified by the projective scaling vector field for this problem using $\mathbf{J}^{-1}$. One can check that this direction field is well defined independent of the choice of $\mathbf{J}$, see part II. A suitable choice for $\mathbf{J}$ is the mapping $\mathbf{y}=\mathbf{J}(\mathbf{x})$ defined by

$$
y_{j}=\frac{\left\langle\mathbf{a}_{j}, \mathbf{x}\right\rangle-b_{j}}{\left\langle\mathbf{a}_{j}, \mathbf{x}_{\mathbf{H}}\right\rangle-b_{j}} ; \quad 1 \leq j \leq m,
$$

where $\mathbf{x}_{H}$ is the center of $\mathrm{H}$.

The second definition uses the notion of centering. Given $x_{0}$ in $\operatorname{Int}\left(P_{\mathrm{H}}\right)$ we choose a projective transformation $\Phi$ with $\Phi\left(\mathbf{x}_{0}\right)=\mathbf{0}$ such that $\mathbf{0}$ is the center of the transformed linear program's constraints. The transformed linear program has constraints $\tilde{H}=\Phi(H)$ and the objective function $\langle\tilde{\mathbf{c}}, \mathbf{x}\rangle-\tilde{c}_{0}$ where 
$\Phi\left(H_{\text {opt }}\right)=\left\{\mathbf{x}:\langle\tilde{\mathbf{c}}, \mathbf{x}\rangle=\tilde{c}_{0}\right\}$ is the transformed optimal solution hyperplane, with sign chosen so that $-\tilde{c}_{0}>0$. Then the projective scaling direction is the ray

$$
\left[\mathbf{v}_{P}(\mathbf{x} ; \mathbf{c}, \mathbf{H})\right]=\left\{\lambda \tilde{\mathbf{v}}_{P}(\mathbf{x} ; \mathbf{c}, \mathbf{H}): \lambda>0\right\}
$$

in the direction of the vector

$$
\tilde{\mathbf{v}}_{P}(\mathbf{x} ; \mathbf{c}, \mathbf{H})=-\left(\Phi^{-1}\right)_{*}\left(\left(\nabla \phi_{\tilde{\mathrm{H}}}(\mathbf{0})\right)^{-1} \tilde{\mathbf{c}} .\right.
$$

Here $\tilde{\mathbf{c}}$ is the transformed objective function direction, $\nabla \phi_{\tilde{\mathrm{H}}}(\mathbf{x})$ is the Jacobian of the Legendre transform mapping $\phi_{\tilde{H}}$. It can be checked that the direction field $\left[\mathbf{v}_{P}(\mathbf{x} ; \mathbf{c}, \mathrm{H})\right]$ is well defined independent of the choice of centering transformation $\Phi$, although the vector $\mathbf{v}_{P}(\mathbf{x} ; \mathbf{c}, \mathrm{H})$ is only well defined up to multiplication by a positive constant, see [BL].

The paper $[\mathrm{BL}]$ shows that these two definitions give the same direction field. That paper also shows that there is a natural definition of a projective scaling vector field, provided that one treats projective transformations algebraically as members of the general linear group $G L(n+1, \mathbf{R})$.

Projective scaling trajectories are the curves obtained by following the projective scaling direction field. We consider them as having a directional orientation in the direction of the direction field. The central P-trajectory $T_{P}(\mathbf{c}, \mathrm{H})$ is the $P$-trajectory through the center $\mathbf{x}_{\mathrm{H}}$ of $P_{\mathrm{H}}$.

Theorem 5.1. Suppose given an inequality form linear program (5.1) having a bounded polytope $P_{\mathrm{H}}$ of feasible solutions with nonempty interior. Any admissible projective transformation for $P_{\mathrm{H}}$ maps $P$-trajectories into $P$-trajectories of the transformed linear program. The central $P$-trajectory $T_{P}(\mathbf{c}, \mathrm{H})$ coincides with the central A-trajectory, and is given by

$$
T_{P}(\mathbf{c}, \mathbf{H})=\left\{\mathbf{x} \in \operatorname{Int}\left(P_{\mathrm{H}}\right): \phi_{\mathbf{H}}(\mathbf{x})=\text { tc for } t \in \mathbf{R}\right\} \text {. }
$$

Proof. Using the first definition, the invariance under projective transformations is proved in part I, Corollary 5.1. Using the centering definition, this invariance is proved in [BL, Theorem 2.5].

The centerl $P$-trajectory and central $A$-trajectory coincide for canonical form linear programs by part II, Theorem 6.1. The central $P$-trajectory and central $A$-trajectory for full-dimensional linear programs (5.1) are both defined (using the first definition) by pulling back by an affine transformation from a canonical form problem, as they are identical. By part II, Theorem 8.1 the central $A$ trajectory is

$$
T_{A}(\mathbf{c}, \mathrm{H})=\left\{\mathbf{x} \in \operatorname{Int}\left(P_{\mathrm{H}}\right): \phi_{\mathrm{H}}(\mathbf{x})=t \mathbf{c} \text { for } t \in \mathbf{R}\right\}
$$

so (5.5) follows.

\section{Projective Legendre transform COORDinAtes LINEARIZE $P$-TRAJECTORIES}

This section considers linear programs in $\mathbf{R}^{n}$ given in inequality form:

$$
\left\{\begin{array}{l}
\operatorname{minimize}\langle\mathbf{c}, \mathbf{x}\rangle-c_{0} \\
\left\langle\mathbf{a}_{j}, \mathbf{x}\right\rangle \geq b_{j} ; \quad 1 \leq j \leq m,
\end{array}\right.
$$


whose objective function $\langle\mathbf{c}, \mathbf{x}\rangle-c_{0}$ is normalized to have optimal value zero. It shows that the projective Legendre transform coordinate mapping linearizes $P$-trajectories. We prove an "affine" version of this result using the polarity correspondence. There is an equivalent coordinate-free "projective" version of the result which we omit.

Theorem 6.1. Suppose given a linear program in $\mathbf{R}^{n}$ in the inequality form (6.1) such that $P_{\mathrm{H}}$ is bounded and $\mathbf{0} \in \operatorname{Int}\left(P_{\mathrm{H}}\right)$. Then the projective Legendre transform mapping maps the P-trajectory through $\mathbf{x}_{0} \in \operatorname{Int}\left(P_{\mathrm{H}}\right)$ onto the straight line segment in $\operatorname{Int}\left(P_{\mathrm{H}}^{\circ}\right)$ passing through the two points $\psi_{\mathrm{H}}\left(\mathbf{x}_{0}\right)$ and $-\mathbf{c} / c_{0}$, where the point $-\mathbf{c} / c_{0}$ corresponds under polarity to the optimal objective function hyperplane $H_{\text {opt }}=\left\{\mathbf{x}:\langle\mathbf{c}, \mathbf{x}\rangle=c_{0}\right\}$.

Proof. We first prove the result in the special case that $\mathbf{x}_{0}=\mathbf{0}$ and $\mathbf{0}$ is the center of $P_{\mathrm{H}}$. By Theorem 5.1 the central $P$-trajectory is

$$
T_{P}(\mathbf{c}, \mathrm{H})=\left\{\mathbf{x} \in \operatorname{Int}\left(P_{\mathrm{H}}\right): \phi_{\mathrm{H}}(\mathbf{x})=t \mathbf{c} \text { for } t \in \mathbf{R}\right\} .
$$

Since the projective Legendre transform mapping

$$
\psi_{H}(\mathbf{x})=\frac{\phi_{H}(\mathbf{x})}{m+\left\langle\phi_{H}(\mathbf{x}), \mathbf{x}\right\rangle}
$$

is a scaled version of $\phi_{H}(\mathbf{x})$ one has

$$
\mathbf{x} \in T_{P}(\mathbf{c}, \mathbf{H}) \Leftrightarrow \psi_{\mathrm{H}}(\mathbf{x})=t^{\prime} \mathbf{c} \quad \text { for some } t^{\prime} \in \mathbf{R} .
$$

Also $\psi_{\mathrm{H}}(\mathbf{0})=\mathbf{0}$ and $-\mathbf{c} / c_{0}$ are on this line, and determine it. This proves the special case.

To prove the general case, observe that there exists a projective transformation $\Phi$ with $\Phi\left(\mathbf{x}_{0}\right)=\mathbf{0}$ such that $\Phi$ centers $\mathbf{x}_{0}$. Theorem 4.1 then gives the following commutative diagram.

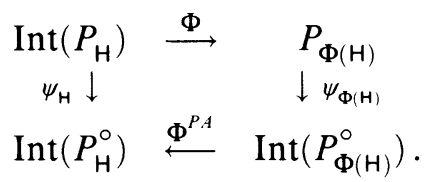

By Theorem 5.1 the $P$-trajectory $T_{P}\left(\mathbf{x}_{0} ; \mathbf{c}, \mathrm{H}\right)$ is mapped by $\Phi$ to the $P$ trajectory $T_{P}(\mathbf{0} ; \tilde{\mathbf{c}}, \Phi(\mathrm{H}))$, where $\Phi\left(H_{\text {opt }}\right)=\left\{\mathbf{y}:\langle\tilde{\mathbf{c}}, \mathbf{y}\rangle=\tilde{c}_{0}\right\}$, and $-\tilde{c}_{0}>0$. By the already proved special case this $P$-trajectory is mapped by $\psi_{\Phi(H)}$ to the segment of the straight line determined by $\psi_{\boldsymbol{\Phi}(\mathrm{H})}(\mathbf{0})$ and $-\tilde{\mathbf{c}} / \tilde{c}_{0}$ lying in $\operatorname{Int}\left(P_{\Phi(\mathrm{H})}^{\circ}\right)$. The projective mapping $\Phi^{P A}$ maps this segment of a straight line to a segment of a straight line in $\operatorname{Int}\left(P_{\mathrm{H}}^{\circ}\right)$, containing $\psi_{\mathrm{H}}\left(\mathbf{x}_{0}\right)$. Finally $-\mathbf{c} / c_{0}$ is a distinct point on this line because $\Phi^{P A}\left(-\tilde{\mathbf{c}} / \tilde{c}_{0}\right)=-\mathbf{c} / c_{0}$, using Theorem 2.6. The general case follows.

The Legendre transform mapping thus maps the set of $P$-trajectories for the normalized objective function $\langle\mathbf{c}, \mathbf{x}\rangle-c_{0}$ to the complete set of line segments in $\operatorname{Int}\left(P_{\mathrm{H}}^{\circ}\right)$ passing through the point $-\mathbf{c} / c_{0}$. The point $\mathbf{c} / c_{0}$ is a boundary point of $P_{\mathrm{H}}^{\circ}$ because $H_{\mathrm{opt}}$ is tangent to $P_{\mathrm{H}}$. (See Figure 1.2.) 
Part I observed that the projective scaling vector field and $P$-trajectories can also be defined for some objective functions that are not normalized. The corresponding full-dimensional version of this definition is to select a particular objective function hyperplane $\hat{H}=\left\{\mathbf{x}:\langle\mathbf{c}, \mathbf{x}\rangle=c_{1}\right\}$ and treat it as "fixed". Then the projective scaling direction field $\left[\mathbf{v}_{P}(\mathbf{x}, \hat{H}, \mathrm{H})\right]$ attached to $\hat{H}$ is defined at $\mathbf{x}_{0}$ by first picking a projective transformation $\Phi$ that centers $\mathbf{x}_{0}$ at $\mathbf{0}$, next defining $\hat{\mathbf{c}}$ by $\Phi(\hat{H})=\left\{\mathbf{x}:\langle\hat{\mathbf{c}}, \mathbf{x}\rangle=\hat{c}_{1}\right\}$ with $-\hat{c}_{1}>0$ and then setting

$$
\mathbf{v}_{P}(\mathbf{x}, \hat{H}, \mathbf{H})=-\left(\Phi^{-1}\right) *\left(\left(\nabla \phi_{\mathrm{H}}(\mathbf{0})\right)^{-1} \hat{\mathbf{c}}\right) .
$$

It can then be shown that under the projective Legendre transform mapping $\psi_{\mathrm{H}}$ the set of associated $P$-trajectories are mapped into the set of straight line segments in $\operatorname{Int}\left(P_{\mathrm{H}}^{\circ}\right)$, whose continuation as a straight line contains the point $-\mathrm{c} / c_{1}$ which corresponds under polarity to the hyperplane $\hat{H}$. The point $-\mathbf{c} / c_{1}$ can be either inside or outside $P_{\mathrm{H}}^{\circ}$. The $P$-trajectories attached to $\hat{H}$ have a directional orientation, as follows. If $c_{1}<0$ then the $P$-trajectories in projective Legendre transform coordinates all point toward $-\mathbf{c} / c_{1}$ and run towards it as $t \rightarrow \infty$, and if $c_{1}>0$ then they point away from $-\mathrm{c} / c_{1}$ and run away from it as $t \rightarrow \infty$. In particular if $-\mathbf{c} / c_{1} \in \operatorname{Int}\left(P_{\mathrm{H}}^{\circ}\right)$ then $\psi_{\mathrm{H}}^{-1}\left(-\mathbf{c} / c_{1}\right)$ is an attractive fixed point (a "sink") of the projective scaling vector field if $c_{1}<0$ and is a repulsive fixed point (a "source") if $c_{1}>0$.

With further analysis one can use Theorem 6.2 to show that the $P$-trajectories for a linear program (6.1) with a normalized objective function viewed in Legendre transform coordinates $\phi_{H}(\mathbf{x})$ become asymptotically parallel to the central $P$-trajectory $\{t \mathbf{c}:-\infty<t<\infty\}$ as $t \rightarrow \infty$.

\section{Hilbert GeOMETRY}

In studying the foundations of geometry, Hilbert posed the question of finding all metrics $d(\cdot, \cdot)$ defined on subsets of $\mathbf{P}^{n}$ such that straight lines segments give the shortest distance between two points, which is the fourth of his wellknown problems [H2]. He had earlier defined a set of metrics, the Hilbert metrics, having this property, [H1, H3, Appendix I]. Let $C$ be a closed convex body on $\mathbf{R}^{n}$. The Hilbert metric $d_{c}$ is defined on $\operatorname{Int}(C)$ by

$$
d_{C}\left(\mathbf{x}_{1}, \mathbf{x}_{2}\right)=\log \left(\frac{\left\|\mathbf{y}_{1}-\mathbf{x}_{2}\right\|}{\left\|\mathbf{y}_{1}-\mathbf{x}_{1}\right\|} \cdot \frac{\left\|\mathbf{y}_{2}-\mathbf{x}_{1}\right\|}{\left\|\mathbf{y}_{2}-\mathbf{x}_{2}\right\|}\right)
$$

where $\mathbf{y}_{1}, \mathbf{y}_{2}$ are the boundary points of the line segment in $C$ defined by $\mathbf{x}_{1}, \mathbf{x}_{2}$, ordered so that $\left(\mathbf{y}_{1}, \mathbf{x}_{1}, \mathbf{x}_{2}, \mathbf{y}_{2}\right)$ are in order on the line segment, and $\|\cdot\|$ is the Euclidean norm. (See Figure 7.1.) The quantity inside the logarithm is the cross-ratio of the points $\left(\mathbf{y}_{1}, \mathbf{x}_{1}, \mathbf{x}_{2}, \mathbf{y}_{2}\right)$.

It can be shown that $d_{C}$ is a metric and that it is invariant under projective transformation $\Phi$ in the sense that

$$
d_{C}\left(\mathbf{x}_{1}, \mathbf{x}_{2}\right)=d_{\Phi(C)}\left(\Phi\left(\mathbf{x}_{1}\right), \Phi\left(\mathbf{x}_{2}\right)\right) ; \quad \mathbf{x}_{1}, \mathbf{x}_{2} \in C,
$$




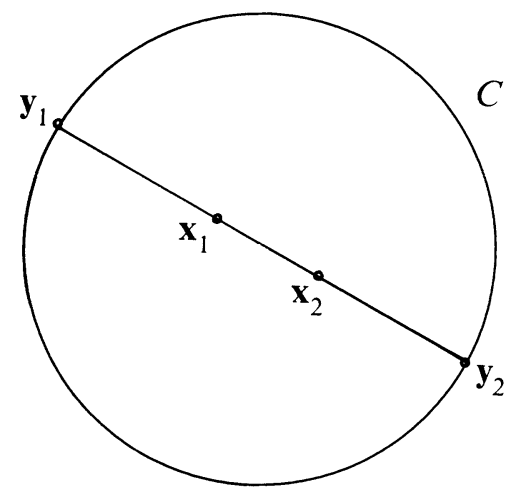

FIGURE 7.1. Hilbert metric

provided $\Phi(C)$ is bounded. A curve is a chord or an extremal if that part of it between any two points $\mathbf{x}_{1}$ and $\mathbf{x}_{2}$ on it is a curve of shortest distance between $\mathbf{x}_{1}$ and $\mathbf{x}_{2}$. If there is a unique extremal connecting $\mathbf{x}_{1}$ and $\mathbf{x}_{2}$, it is called a geodesic. Straight line segments in $\operatorname{Int}(C)$ are chords for the Hilbert metric. If $C$ is strictly convex, then all such straight line segments are geodesics. If $C$ is a polytope, then extremals are not always unique, so that geodesics are not defined. Busemann [Bu1, BP] has shown that one can sometimes obtain reasonable geometries, which he calls chord geometries, by treating a subset of the extremals as "geodesics", calling them distinguished chords. In particular when $C$ is a polytope, taking the set of straight line segments in $\operatorname{Int}(C)$ as distinguished chords gives a chord geometry in Busemann's sense. Hilbert geometry is the geometry on $\operatorname{Int}(C)$ induced by the Hilbert metric with straight line segments taken as distinguished chords. Hilbert geometry is complete in the sense that distinguished chords are of infinite length in any direction in the Hilbert metric.

The Hilbert metric behaves in many aspects like a metric of constant negative curvature. In fact if $C$ is an ellipsoid then the Hilbert metric gives the usual hyperbolic metric and geometry on the interior of the ellipsoid. Hilbert geometry has constant negative curvature in the sense of Berwald (see [Bu2, p. 139]).

The projective Legendre transform coordinate mapping yields a natural interpretation of the complete set $P$-trajectories (for all objective functions) as the set of distinguished chords of a metric $d_{\mathrm{H}}(\cdot, \cdot): \operatorname{Int}\left(P_{\mathrm{H}}\right) \times \operatorname{Int}\left(P_{\mathrm{H}}\right) \rightarrow \mathbf{R}$. This metric is the pullback under $\psi_{\mathrm{H}}^{-1}$ of the Hilbert metric on the interior of the dual polytope $\operatorname{Int}\left(P_{\mathrm{H}}^{d}\right)$, i.e.

$$
d_{\mathrm{H}}\left(\mathbf{x}_{1}, \mathbf{x}_{2}\right) \equiv d_{P_{\mathrm{H}}^{d}}\left(\psi_{\mathrm{H}}\left(\mathbf{x}_{1}\right), \psi_{\mathrm{H}}\left(\mathbf{x}_{2}\right)\right) .
$$

We have the following result.

Theorem 7.1. Given a set $\mathrm{H}$ of inequality constraints on $\mathbf{R}^{n}$ :

$$
\left\langle\mathbf{a}_{j}, \mathbf{x}\right\rangle \geq b_{j} ; \quad 1 \leq j \leq m
$$


such that $P_{\mathrm{H}}$ is bounded with nonempty interior. The P-trajectories for all objective functions make up a complete set of distinguished chords for the metric $d_{\mathrm{H}}$ on $\operatorname{Int}\left(P_{\mathrm{H}}\right)$. If $\Phi$ is an admissible projective transformation for $\mathrm{H}$ then

$$
d_{\mathrm{H}}\left(\mathbf{x}_{1}, \mathbf{x}_{2}\right)=d_{\Phi(\mathrm{H})}\left(\Phi\left(\mathbf{x}_{1}\right), \Phi\left(\mathbf{x}_{2}\right)\right) .
$$

Proof. This is an immediate consequence of Theorem 6.1.

$P$-trajectories can equally well be interpreted as "geodesics" of a projectively invariant metric obtained as the pullback of any projectively invariant metric on $P_{\mathrm{H}}^{d}$ such that straight line segments are chords. Any such metric provides a projective invariant for measuring the progress of interior-point linear programming algorithms. The Hilbert metric may possibly be useful in obtaining improved running time bounds for Karmarkar's algorithm.

\section{PRojective DUAlity AND LiNEAR PROgRAMMing DUALITY}

There is a relation between projective duality and the ordinary duality theory of linear programming.

Consider a linear program on $\mathbf{R}^{n}$ given in the inequality form:

$$
(P)\left\{\begin{array}{l}
\operatorname{minimize}\langle\mathbf{c}, \mathbf{x}\rangle \\
A \mathbf{x} \geq \mathbf{b},
\end{array}\right.
$$

where $\mathbf{A}=\left[\mathbf{a}_{1}, \ldots, \mathbf{a}_{m}\right]^{T}$, and suppose that $\mathbf{0}$ is an interior feasible point of $(P)$. The linear program $(D)$ dual to $(P)$ is

$$
\text { (D) }\left\{\begin{array}{l}
\text { minimize }-\langle\mathbf{b}, \mathbf{y}\rangle \\
A^{T} \mathbf{y}=\mathbf{c}, \\
\mathbf{y} \geq \mathbf{0} .
\end{array}\right.
$$

Let $P_{(P)}, P_{(D)}$ denote the polytope of feasible solutions to $(\mathrm{P})$ and $(\mathrm{D})$ respectively, and let $P_{(P)}^{\circ}$ denote the polytope polar to $P_{(P)}$. The polar polytope $P_{(P)}^{\circ}$ is the convex hull of the points $\mathbf{a}_{1} / b_{1}, \mathbf{a}_{2} / b_{2}, \ldots, \mathbf{a}_{n} / b_{n}$, so that

$$
P_{(P)}^{\circ}=\left\{\lambda: \lambda=\sum_{l=1}^{m} \lambda_{i} \frac{1}{b_{i}} \mathbf{a}_{i}, \text { all } \lambda_{i} \geq 0, \sum_{i=1}^{m} \lambda_{i}=1\right\} .
$$

Since the linear program $(P)$ has full rank, $P_{(P)}^{\circ}$ is a full-dimensional polytope and

$$
\operatorname{Int}\left(P_{(P)}^{\circ}\right)=\left\{\lambda: \lambda=\sum_{l=1}^{m} \lambda_{i} \frac{1}{b_{i}} \mathbf{a}_{i}, \text { all } \lambda_{i}>0, \sum_{i=1}^{m} \lambda_{i}=1\right\} .
$$

The dual linear program polytope $P_{(D)}$ depends on the objective function $\langle\mathbf{c}, \mathbf{x}\rangle$ of $(P)$, while the polar polytope $P_{(P)}^{\circ}$ does not. There is however a natural projection map $\pi_{P, D}: \operatorname{Rel}-\operatorname{Int}\left(P_{(D)}\right) \rightarrow \operatorname{Int}\left(P_{(P)}^{\circ}\right)$ defined by

$$
\pi_{P, D}(\mathbf{y})=\sum_{j=1}^{m} \frac{1}{b_{j}} \mathbf{a}_{j}\left(\frac{b_{j} y_{j}}{\langle\mathbf{b}, \mathbf{y}\rangle}\right) .
$$


This map is neither one-to-one nor onto, in general.

Let $\mathrm{H}_{P}$ denote the constraints of $(P)$, and $\mathrm{H}_{D}$ the constraints of $(D)$. We have shown that the projective Legendre transform coordinate mapping $\psi_{\mathrm{H}}(\mathbf{x})$ gives a one-to-one and onto rational map from $\operatorname{Int}\left(P_{(P)}\right)$ to $\operatorname{Int}\left(P_{(P)}^{\circ}\right)$. In general there is no one-to-one algebraic map from $\operatorname{Int}\left(P_{(P)}\right)$ to $\operatorname{Rel-Int}\left(P_{(D)}\right)$, because the dimensions of $P_{(P)}$ and $P_{(D)}$ are usually different. However in part II, $\S 10$, we showed that if $\mathbf{c} \neq \mathbf{0}$ then there is a rational map $\delta^{(P)}$ from the positive part $T_{+}^{(P)}$ of the central trajectory for $(P)$, which is defined by

$$
T_{+}^{(P)}=\left\{\mathbf{x}(t): \phi_{\mathrm{H}_{P}}(\mathbf{x}(t))=-t \mathbf{c}, 0<t<\infty\right\},
$$

to the positive part $T_{+}^{(D)}$ of the central trajectory for the dual linear program $(D)$, which is defined by

$$
T_{+}^{(D)}=\left\{\mathbf{y}(t): \phi_{\mathbf{H}_{D}}(\mathbf{y}(t))=t \pi_{A^{\perp}}(\mathbf{b}), 0<t<\infty\right\} .
$$

The map $\boldsymbol{\delta}^{(P)}$ satisfies

$$
\boldsymbol{\delta}^{(P)}(\mathbf{x}(t))=\mathbf{y}(t)
$$

and is given explicitly by

$$
\boldsymbol{\delta}^{(P)}(\mathbf{x}(t))_{j}=\frac{1}{t\left(\left\langle\mathbf{a}_{j}, \mathbf{x}\right\rangle-b_{j}\right)} ; \quad 1 \leq j \leq m .
$$

The map $\delta^{(P)}$ is a one-to-one mapping from the subset $T_{+}^{(P)}$ of $\operatorname{Int}\left(P_{(P)}\right)$ to the subset $T_{+}^{(D)}$ of $\operatorname{Rel-Int}\left(P_{(D)}\right)$.

Theorem 8.1. Let $(P)$ and $(D)$ be a pair of dual linear programs such that $\mathbf{0}$ is an interior feasible point of $(P)$. If $\mathbf{x}(t)$ is on the positive part $T_{+}^{(P)}$ of the central trajectory of $(P)$ then $\boldsymbol{\delta}^{(P)}(\mathbf{x}(t))$ is in $\operatorname{Rel}-\operatorname{Int}\left(P_{(D)}\right)$ and

$$
\psi_{\mathrm{H}}(\mathbf{x}(t))=\pi_{P, D}\left(\boldsymbol{\delta}^{(P)}(\mathbf{x}(t))\right)
$$

Proof. This is a simple computation. Let $\mathbf{x}=\mathbf{x}(t)$ and $\mathbf{y}=\mathbf{y}(t)$ and using (8.1)-(8.3) we have

$$
\pi_{P, D}\left(\boldsymbol{\delta}^{(P)}(\mathbf{x})\right)=\sum_{j=1}^{n} \frac{1}{b_{j}} \mathbf{a}_{j}\left(\frac{b_{j} y_{j}}{\langle\mathbf{b}, \mathbf{y}\rangle}\right)=-\frac{1}{t\langle\mathbf{b}, \mathbf{y}\rangle} \phi_{\mathrm{H}}(\mathbf{x}) .
$$

Now

$$
\begin{aligned}
-\frac{1}{t\langle\mathbf{b}, \mathbf{y}\rangle} & =-\frac{1}{\sum_{j=1}^{m} \frac{b_{j}}{\left\langle\mathbf{a}_{j}, \mathbf{x}\right\rangle-b_{j}}}=\frac{1}{m-\sum_{j=1}^{m} \frac{\left\langle\mathbf{a}_{j}, \mathbf{x}\right\rangle}{\left\langle\mathbf{a}_{j}, \mathbf{x}\right\rangle-b_{j}}} \\
& =\frac{1}{m+\left\langle\phi_{\mathbf{H}}(\mathbf{x}), \mathbf{x}\right\rangle} .
\end{aligned}
$$

Hence the right side of $(8.5)$ is $\psi_{H}(\mathbf{x})$ and the theorem follows. 
To summarize: The projective Legendre transform coordinate mapping gives a one-to-one correspondence between the interior of a feasible solution polytope $P_{(P)}$ and the interior of its polar (projectively dual) polytope $P_{(P)}^{\circ}$. There is no algebraic correspondence in general between the interior of $P_{(P)}$ and its linear programming dual polytope $P_{(D)}$ but there is a (rational) correspondence between central trajectories in $P_{(P)}$ and $P_{(D)}$, and this correspondence is compatible with the projective Legendre transform coordinate mapping.

\section{REFERENCES}

[A] K. Ansteicher, Linear programming and the Newton barrier flow, Math. Programming 41 (1988), 367-374.

[BL1] D. Bayer and J. C. Lagarias, The nonlinear geometry of linear programming. I. Affine and projective scaling trajectories, Trans. Amer. Math. Soc. 314 (1989), 499-526.

[BL2] _ - The nonlinear geometry of linear programming. II. Legendre transform coordinates and central trajectories, Trans. Amer. Math. Soc. 314 (1989), 527-581.

[BL] _- Karmarkar's linear programming algorithm and Newton's method, Math. Programming (to appear).

[Bi] G. Birkhoff, Extensions of Jentzch's theorem, Trans. Amer. Math. Soc. 85 (1957), 219-227.

[Bu1] H. Busemann, The geometry of geodesics, Academic Press, New York, 1955.

[Bu2] _ Spaces with distinguished shortest joins, Mathematical Developments Arising from Hilbert Problems, Proc. Sympos. Pure Math., vol. 28, Amer. Math. Soc., Providence, R.I., 1976, pp. 131-142.

[BP] H. Busemann and B. B. Phadke, Beltrami's theorem in the large, Pacific J. Math. 115 (1984), 299-315.

[Bush] P. Bushnell, On the projective contraction ratio for positive linear mappings, J. London Math. Soc. 6 (1973), 256-258.

[Bush2] __, Hilbert's metric and positive contraction mappings in a Banach space, Arch. Rational Math. Anal. 52 (1973), 330-338.

[Fe1] W. Fenchel, A remark on convex sets and polarity, Comm. Sém. Math. Univ. Lund, Tome Suppl., 1952, pp. 82-89.

[Fe2] _ Convex cones, sets, and functions, Mimeographed Lecture Notes, Princeton Univ., 1953.

[H1] D. Hilbert, Ueber die gerade Linie als Kürzeste Verbindung zwier punkte, Math. Ann. 46 (1895), 91-96 (Appendix I of [H3]).

[H2] _- Mathematical problems, Bull. Amer. Math. Soc. 8 (1902), 437-479. (Transl. of Mathematische Probleme, Gottinger Nachrichten, 1900, pp. 253-297).

[H3] _ Foundations of geometry, 10th ed. (translated by Leo Ungar), Open Court, La Salle, Ill., 1971.

[K] N. Karmarkar, A new polynomial time algorithm for linear programming, Combinatorica 4 (1984), 373-395.

[K1] V. Klee, Adjoints of projective transformations and face figures of convex polytopes, Math. Programming Stud. 3 (1978) 208-216.

[Ko] S. Kobayashi, Invariant distances for projective structures, Sympos. Math. 26 (1982), $153-$ 161.

[KP] E. Kohlberg and J. W. Pratt, The contraction mapping approach to the Perron-Frobenius theory: Why Hilbert's metric?, Math. Oper. Res. 7 (1982), 198-250.

[MS] N. Megiddo and M. Shub, Boundary behavior of interior point algorithms in linear programming, Math. Oper. Res. 14 (1989), 97-146. 
[Sh] M. Shub, On the asymptotic behavior of the projective rescaling algorithm for linear programming, IBM Report RC 12522, 1987.

[S1] F. Sinden, A geometric representation for pairs of dual quadratic or linear programs, J. Math. Anal. Appl. 5 (1962), 378-402.

[S2] _ Duality in convex programming and in projective space, J. Soc. Indust. Appl. Math. 11 (1963), 535-552.

[Sol] G. Sonnevend, An "analytical centre" for polyhedrons and new classes of global algorithms for linear (smooth, convex) programming, Proc. 12th IFIP Conf. System Modelling and Optimization 1985, Lecture Notes in Control and Information Science Vol. 84, SpringerVerlag, New York, 1986.

[So2] _ A new method of solving a set of linear (convex) inequalities and its application for identification and optimization, Proc. Sympos. Dynamic Modelling, IFAC-IFORS, Budapest, 1986.

[St] E. Steinitz, Bedingt konvergente Reihen und konvexe Systeme. I, II, III, J. Reine Angew Math. 143 (1913), 128-195; 144 (1914), 1-40: 146 (1916), 1-52.

AT\&T Bell Laboratories, Murray Hill, New Jersey 07974 